Journal of Mathematical Physics, Analysis, Geometry

2016, vol. 12, No. 1, pp. 48-77

\title{
Solvability in Hölder Space of an Initial Boundary Value Problem for the Time-Fractional Diffusion Equation
}

\author{
M.V. Krasnoschok \\ Institute of Applied Mathematics and Mechanics \\ National Academy of Sciences of Ukraine \\ 1 Dobrovol'skogo Str., Slov'yansk 84100, Ukraine \\ E-mail: iamm012@ukr.net
}

Received October 13, 2014, revised April 20, 2015, published online December 4, 2015

In this paper we consider an initial boundary value problem for the timefractional diffusion equation with mixed boundary conditions. We prove a theorem of existence and uniqueness of solution to this problem in Hölder spaces.

Key words: fractional-order diffusion, Hölder spaces, Caputo derivative.

Mathematics Subject Classification 2010: 35B65 (primary); 26A33 (secondary).

\section{Introduction}

Let $\partial_{t}^{\alpha}(0<\alpha<1)$ be a regularized fractional derivative (the Caputo derivative), that is,

$$
\partial_{t}^{\alpha} u(x, t)=\frac{1}{\Gamma(1-\alpha)}\left[\frac{\partial}{\partial t} \int_{0}^{t}(t-\tau)^{-\alpha} u(x, \tau) d \tau-t^{-\alpha} u(x, 0)\right] .
$$

Let $\Omega$ be a doubly connected bounded domain in $\mathbb{R}^{n}$ with the boundary $\partial \Omega=\Sigma^{1} \cup \Sigma^{2}\left(\Sigma^{1} \cap \Sigma^{2}=\varnothing\right)$. Denote $\Omega_{T}=\Omega \times(0, T), \Sigma_{T}^{i}=\Sigma^{i} \times(0, T), i=1,2$, $T>0$.

We need to find the function $u(x, t)$ satisfying the equation

$$
\mathcal{L}\left(x, t, \frac{\partial}{\partial x}, \partial_{t}^{\alpha}\right) u=f(x, t), \quad \text { in } \quad \Omega_{T},
$$

(c) M.V. Krasnoschok, 2016 
with the initial and boundary conditions

$$
\begin{gathered}
\left.u\right|_{t=0}=u_{0}(x), \quad x \in \Omega, \\
\left.u\right|_{\Sigma^{1}}=\psi_{1}(x, t),\left.\quad \mathcal{B}\left(x, t, \frac{\partial}{\partial x}\right) u\right|_{\Sigma^{2}}=\psi_{2}(x, t), \quad t \in(0, T),
\end{gathered}
$$

where

$$
\begin{aligned}
\mathcal{L}\left(x, t, \frac{\partial}{\partial x}, \partial_{t}^{\alpha}\right)= & \partial_{t}^{\alpha}-\sum_{i, j=1}^{n} a_{i j}(x, t) \frac{\partial^{2}}{\partial x_{i} \partial x_{j}}-\sum_{i=1}^{n} a_{i}(x, t) \frac{\partial}{\partial x_{i}}-a_{0}(x, t), \\
& \mathcal{B}\left(x, t, \frac{\partial}{\partial x}\right)=\sum_{i=1}^{n} b_{i}(x, t) \frac{\partial}{\partial x_{i}}+b_{0}(x, t) .
\end{aligned}
$$

We assume

$$
\begin{gathered}
\nu \xi^{2} \leq \sum_{i, j=1}^{n} a_{i j}(x, t) \xi_{i} \xi_{j} \leq \mu \xi^{2} \quad \text { for all }(x, t) \in \Omega_{T}, \\
\sum_{i=1}^{n} b_{i}(x, t) n_{i}(x) \leq-\delta<0 \quad \text { for all }(x, t) \in \Sigma_{T}^{2}
\end{gathered}
$$

where $n(x)$ is the unit outward normal to $\Sigma^{2}$ at the point $x$.

Fractional diffusion equations were applied to numerous problems in biology, chemistry, hydrology, etc. (see, for example, [1, 14, 23], Chapter 10 in [17] and Chapter 5 in [16]).

In [9], Kochubei found the expression for the fundamental solution $\Gamma_{\alpha}(x, t)$ to the Cauchy problem

$$
\begin{gathered}
\partial_{t}^{\alpha} u-\Delta u=f(x, t), \quad(x, t) \in \mathbb{R}^{n} \times(0, T), \\
u(x, 0)=u_{0}(x), \quad x \in \mathbb{R}^{n},
\end{gathered}
$$

in terms of Fox's H-functions (see also [22]). In [19], Pskhu represented $\Gamma_{\alpha}(x, t)$ using Wright functions

$$
\phi(-\alpha, \delta ; z)=\sum_{k=0}^{\infty} \frac{z^{k}}{k ! \Gamma(\delta-\alpha k)}, \quad \beta \in(0,1) .
$$

Besides, some estimates of $\Gamma_{\alpha}(x, t)$ and its derivatives are derived in $[9,19]$.

The Cauchy problem for the operator $\mathcal{L}\left(x, t, \frac{\partial}{\partial x}, \partial_{t}^{\alpha}\right)$ (see (1.5)) and fractional order parabolic systems were studied in $[3,10]$. 
The papers $[4,15,21,24]$ give the results on solvability of some initial boundary value problems for linear and quasilinear fractional diffusion equations in Sobolev spaces.

H. Lopushans'ka and A. Lopushans'kyi [13] proved the theorem on the existence and uniqueness of the solution to the space-time fractional Cauchy problem in spaces of generalized functions.

Kemppainen used in [6] the boundary integral approach to prove the existence of the solution for a time-fractional diffusion equation with Robin boundary condition in the space of continuous up to the boundary functions.

Clément, Londen, Simonett obtained in [2] the existence, uniqueness, and continuaty on quasilinear parabolic equation with time-fractional derivative. They considered this derivative in spaces of continuous functions having a prescribed singularity as $t \rightarrow 0$.

In [18], R. Ponce described the well-posedness (or maximal regularity) of the abstract fractional differential equation $(\beta>0)$,

$$
\partial_{t}^{\beta} u(t)=A u(t)+\int_{-\infty}^{t} a(t-s) A u(s) d s+f(t), t \in \mathbb{R},
$$

in $C^{\gamma}(\mathbb{R} ; X), \gamma \in(0,1)$, where $A$ is a closed linear operator defined on a Banach space $X$.

The aim of this paper is to extend classical theory [12] to the case of the problem of fractional order and to prove solvability of problem (1.2)-(1.4) in Hölder spaces. We consider the case $n \geq 2$. The one-dimensional case was studied in [11].

The paper is organized as follows. In Sec. 2, we define the functional spaces and state the main result. The estimates of solutions of model problems are given in Sec. 3. In Sec. 4 we prove the main result. Appendix contains the proof of some auxiliary assertions used in Sec. 3.

\section{Hölder Spaces and the Main Result}

Let us define the Hölder spaces used in this paper. Let $Q$ be a domain in $\mathbb{R}^{n}$, $Q_{T}=Q \times(0, T), \theta \in(0,1)$.

By $C_{\alpha}^{\theta}\left(Q_{T}\right)$, we mean the set of functions defined in $Q_{T}$ and having a finite norm

$$
|w|_{\alpha, Q_{T}}^{(\theta)}=|w|_{Q_{T}}+\langle w\rangle_{\alpha, Q_{T}}^{(\theta)},
$$

where

$$
\begin{aligned}
|w|_{Q_{T}} & =\sup _{(x, t) \in Q_{T}}|w(x, t)|, \\
\langle w\rangle_{\alpha, Q_{T}}^{(\theta)} & =\langle w\rangle_{x, Q_{T}}^{(\theta)}+\langle w\rangle_{t, Q_{T}}^{\left(\frac{\theta \alpha}{2}\right)},
\end{aligned}
$$




$$
\begin{aligned}
& \langle w\rangle_{x, Q_{T}}^{(\theta)}=\sup _{x, y \in Q} \sup _{t \in(0, T)}|w(x, t)-w(y, t)||x-y|^{-\theta}, \\
& \langle w\rangle_{t, Q_{T}}^{(\theta)}=\sup _{x \in Q} \sup _{t, \tau \in(0, T)}|w(x, t)-w(x, \tau)||t-\tau|^{-\theta} .
\end{aligned}
$$

Let $D_{x}^{l}=\frac{\partial^{|l|}}{\partial x_{1}^{l_{1}} \ldots \partial x_{n}^{l_{n}}}, l=\left(l_{1}, \ldots, l_{n}\right),|l|=l_{1}+\ldots+l_{n} . \quad \operatorname{By} C_{\alpha}^{k+\theta}\left(Q_{T}\right)$, $(k=1,2)$, we mean the set of functions $w(x, t)$ with a finite norm $(m=0,1)$,

$$
\begin{gathered}
|w|_{\alpha, Q_{T}}^{(k+\theta)}=\sum_{|l|+2 m \leq k}\left|\partial_{t}^{\alpha m} D_{x}^{l} w\right|_{Q_{T}}+\langle w\rangle_{\alpha, Q_{T}}^{(k+\theta)}, \\
\langle w\rangle_{\alpha, Q_{T}}^{(k+\theta)}=\sum_{|l|+2 m=k}\left\langle\partial_{t}^{\alpha m} D_{x}^{l} w\right\rangle_{\alpha, Q_{T}}^{\theta}+\sum_{|l|=k-1}\left\langle D_{x}^{l} w\right\rangle_{t, Q_{T}}^{\frac{k-1+\theta}{2}} \alpha
\end{gathered}
$$

By the symbol $C_{\alpha, 0}^{k+\theta}\left(Q_{T}\right)$, we denote the subspace of $C_{\alpha}^{k+\theta}\left(Q_{T}\right),(k=0,1,2)$ consisting of functions $w(x, t)$ such that $\left.\partial_{t}^{\alpha m} u\right|_{t=0}=0$, where $m=0$ for $k=0,1$ and $m=0,1$ for $k=2$. With the help of local coordinates and partition of unity, all these spaces can be introduced on manifolds $\Sigma_{T}^{1}, \Sigma_{T}^{2}$.

If $\alpha=1$, then the spaces $C_{\alpha}^{k+\theta}\left(Q_{T}\right)$ coincide with the classical Hölder spaces $H^{k+\theta, \frac{k+\theta}{2}}\left(Q_{T}\right)$ (see Chapter I in [12]).

Now we assume that the compatibility conditions

$$
\begin{gathered}
\psi_{1}(x, 0)=u_{0}(x), \quad \partial_{t}^{\alpha} \psi_{1}(x, 0)=\sum_{i, j=1}^{n} a_{i j}(x, 0) \frac{\partial^{2} u_{0}(x)}{\partial x_{i} \partial x_{j}}+\sum_{i=1}^{n} a_{i}(x, 0) \frac{\partial u_{0}(x)}{\partial x_{i}} \\
+a_{0}(x, 0) u_{0}(x), x \in \Sigma^{1}, \quad \mathcal{B}\left(x, 0, \frac{\partial}{\partial x}\right) u_{0}(x)=\psi_{2}(x, 0), x \in \Sigma^{2}
\end{gathered}
$$

hold.

The main result of this paper is the following.

Theorem 2.1. Suppose that

$$
\Sigma^{1}, \Sigma^{2} \in C^{2+\theta}, \quad a_{i j}, a_{i}, a_{0} \in C_{\alpha}^{\theta}\left(\Omega_{T}\right), b_{i}, b_{0} \in C_{\alpha}^{1+\theta}\left(\Sigma_{T}^{1}\right) i, j=1, \ldots, n,
$$

and assumptions (1.6), (1.7), (2.1) hold. Then for every function $u_{0} \in C^{2+\theta}(\Omega)$, $f \in C_{\alpha}^{\theta}\left(\Omega_{T}\right), \psi_{1} \in C_{\alpha}^{2+\theta}\left(\Sigma_{T}^{1}\right), \psi_{2} \in C_{\alpha}^{1+\theta}\left(\Sigma_{T}^{2}\right)$, problem (1.2)-(1.4) has a unique solution $u \in C_{\alpha}^{2+\theta}\left(\Omega_{T}\right)$ satisfying the estimate

$$
|u|_{\alpha, \Omega_{T}}^{(2+\theta)} \leq C\left(\left|u_{0}\right|_{\Omega}^{(2+\theta)}+|f|_{\alpha, Q_{T}}^{(\theta)}+\left|\psi_{1}\right|_{\alpha, \Sigma_{T}^{1}}^{(2+\theta)}+\left|\psi_{2}\right|_{\alpha, \Sigma_{T}^{2}}^{(1+\theta)}\right) .
$$


Further, let us recall that

$$
I_{t}^{\gamma} v(x, t)=\frac{1}{\Gamma(\gamma)} \int_{0}^{t} \frac{v(x, \tau)}{(t-\tau)^{1-\gamma}} d \tau
$$

is the Riemann-Liouville fractional integral of order $\gamma>0$, and

$$
D_{t}^{\gamma} v(x, t)=\frac{\partial^{k}}{\partial t^{k}} I_{t}^{k-\gamma} v(x, t), \quad k-1<\gamma \leq k, k \in \mathbb{N},
$$

is the Riemann-Liouville fractional derivative of order $\gamma$ (see Chapter 2 in [8]).

We put

$$
I_{t}^{0} v(x, t)=v(x, t), \quad D_{t}^{\zeta} v(x, t)=I_{t}^{-\zeta} v(x, t) \quad \text { if } \quad \zeta<0 .
$$

It can easily be checked that

$$
\partial_{t}^{\alpha} v(x, t)=D_{t}^{\alpha}(v(x, t)-v(x, 0)) .
$$

The properties of Wright functions $\phi(-\alpha, \delta, z)$ are described in detail, for example, in $[19,20,7]$. We put conventionally in $(1.10)$ that $\frac{1}{\Gamma(-k)}=0$ if $k \in$ $\mathbb{N} \cup 0$.

Lemma 3.3.1 in [20] implies

$$
\phi(-\alpha, \delta,-z)>0 \text { if } z>0, \delta \geq 0 .
$$

To this end, we prove the following auxiliary result.

Lemma 2.1. Let $\delta \in \mathbb{R}$. The Wright function $\phi(-\alpha, \delta,-z)$ with $z>0$ satisfies the inequalities

$$
|\phi(-\alpha,-\delta,-z)| \leq c \exp \left(-\sigma z^{\frac{1}{1-\alpha}}\right)\left\{\begin{array}{l}
1, \text { if } \delta \notin \mathbb{N} \bigcup 0, \\
z, \text { if } \delta \in \mathbb{N} \bigcup 0 .
\end{array}\right.
$$

P r o o f. The estimate

$$
|\phi(-\alpha, \nu,-z)| \leq c \exp \left(-\sigma z^{\frac{1}{1-\alpha}}\right) \text { for all } \nu \in \mathbb{R}
$$

follows immediately from Lemmas 2, 3 in [19], and thus inequality (2.9) for $\delta \notin$ $\mathbb{N} \bigcup 0$ is proved.

For $\delta \in \mathbb{N} \bigcup 0$, we use the formula (see (2.2.5) in [20])

$$
\phi(-\alpha,-k,-z)=\alpha z \phi(-\alpha,-k+1-\alpha,-z)-k \phi(-\alpha,-k+1,-z) .
$$

By using this formula and the method of induction, one can easily prove

$$
\phi(-\alpha,-k,-z)=\alpha z \sum_{m=0}^{k}(-1)^{k-m} \frac{k !}{m !} \phi(-\alpha,-m+1-\alpha,-z) .
$$

Combining (2.10), (2.12), we obtain (2.9) for $\delta \in \mathbb{N} \bigcup 0$. The proof is finished. 


\section{Model Problems}

An important role in the proof of Theorem 2.1 is played by the estimates of the solutions to the following three model problems. Firstly, we determine the function $u(x, t)$ as the solution to the Cauchy problem (1.8), (1.9). Secondly, we find the function $v(x, t)$ as the solution of the first boundary value problem in the half-space $\mathbb{R}_{+}^{n}=\left\{x \in \mathbb{R}^{n}: x_{n}>0\right\}$ :

$$
\begin{gathered}
\partial_{t}^{\alpha} v-\Delta v=0,(x, t) \in \mathbb{R}_{+, T}^{n}=\mathbb{R}_{+}^{n} \times(0, T), \\
v(x, 0)=0, x \in \mathbb{R}^{n}, \quad v \rightarrow 0 \text { with }|x| \rightarrow \infty, \\
v\left(x^{\prime}, 0, t\right)=\Phi\left(x^{\prime}, t\right), x^{\prime}=\left(x_{1}, \cdots, x_{n-1}\right) \in \mathbb{R}^{n-1} .
\end{gathered}
$$

Thirdly, we find the function $w(x, t)$ as the solution of the oblique derivative boundary value problem

$$
\begin{gathered}
\partial_{t}^{\alpha} w-\Delta w=0, \quad(x, t) \in \mathbb{R}_{+, T}^{n}=\mathbb{R}_{+}^{n} \times(0, T), \\
w(x, 0)=0, x \in \mathbb{R}^{n}, \quad w \rightarrow 0 \text { with }|x| \rightarrow \infty, \\
\sum_{i=1}^{n} h_{i} \frac{\partial w}{x_{i}}\left(x^{\prime}, 0, t\right)=\Psi\left(x^{\prime}, t\right), x^{\prime}=\left(x_{1}, \cdots, x_{n-1}\right) \in \mathbb{R}^{n-1} .
\end{gathered}
$$

Here $h=\left(h_{1}, \ldots, h_{n}\right)$ is a constant vector.

We assume that

$$
\begin{gathered}
u_{0} \in C^{2+\theta}\left(\mathbb{R}^{n}\right), f \in C_{\alpha}^{\theta}\left(\mathbb{R}_{T}^{n}\right) ; \\
u_{0}(x), f(x, t)=0 \text { for all }|x|>R>0, \\
\Phi \in C_{\alpha, 0}^{2+\theta}\left(\mathbb{R}_{T}^{n-1}\right), \Psi \in C_{\alpha, 0}^{1+\theta}\left(\mathbb{R}_{T}^{n-1}\right) ; \\
\Phi\left(x^{\prime}, t\right)=0, \quad \Psi\left(x^{\prime}, t\right)=0 \text { for all }\left|x^{\prime}\right|>R>0 .
\end{gathered}
$$

Owing to (3.4), we can take

$$
\Phi\left(x^{\prime}, t\right)=0, \quad \Psi\left(x^{\prime}, t\right)=0, \quad t \leq 0 .
$$

Lemma 3.1. Let assumptions (3.3) hold. Then the solution of (1.8), (1.9) is represented as

$$
\begin{gathered}
u(x, t)=\left(\Gamma_{\alpha} * f\right)+\left(D_{t}^{\alpha-1} \Gamma_{\alpha} *_{1} u_{0}\right) \\
\equiv \int_{0}^{t} \int_{\mathbb{R}^{n}} \Gamma_{\alpha}(x-y, t-\tau) f(y, \tau) d y+\int_{\mathbb{R}^{n}} D_{t}^{\alpha-1} \Gamma_{\alpha}(x-y, t) u_{0}(y) d y, \\
\text { where } \Gamma_{\alpha}(x, t)=(4 \pi)^{-\frac{n}{2}} \int_{0}^{\infty} \lambda^{-\frac{n}{2}} \exp \left(-\frac{|x|^{2}}{4 \lambda}\right) t^{-1} \phi\left(-\alpha, 0,-\lambda t^{-\alpha}\right) d \lambda .
\end{gathered}
$$


$\mathrm{P}$ r o o f. We take the Fourier transform in the spatial variables $x$ and the Laplace transform in $t$,

$$
\begin{gathered}
F[u]=\int_{\mathbb{R}^{n}} v(x, t) \exp (-i x \cdot \xi) d x, \quad \xi=\left(\xi_{1}, \ldots, \xi_{n}\right), \\
L[u]=\int_{0}^{\infty} u(x, t) \exp (-p t) d t .
\end{gathered}
$$

Problem (1.8), (1.9) then reduces to the relation (see (2.253) in [17]):

$$
p^{\alpha} \widetilde{u}-p^{\alpha-1} F\left[u_{0}\right]+|\xi|^{2} \widetilde{u}=\widetilde{f},
$$

here $\widetilde{u}=F[L[u]]$. By (3.8), we have

$$
\begin{gathered}
\widetilde{u}=\frac{\tilde{f}}{p^{\alpha}+|\xi|^{2}}+\frac{p^{\alpha-1}}{p^{\alpha}+|\xi|^{2}} F\left[u_{0}\right] \\
=\int_{0}^{\infty} \exp \left(-\left(p^{\alpha}+|\xi|^{2}\right) \lambda\right) d \lambda \widetilde{f}+\int_{0}^{\infty} p^{\alpha-1} \exp \left(-\left(p^{\alpha}+|\xi|^{2}\right) \lambda\right) d \lambda F\left[u_{0}\right] .
\end{gathered}
$$

Combining

$$
\begin{gathered}
F^{-1}\left[\exp \left(-|\xi|^{2} \lambda\right)\right]=(4 \pi \lambda)^{-\frac{n}{2}} \exp \left(-\frac{|x|^{2}}{4 \lambda}\right), \\
L^{-1}\left[p^{-\mu} \exp \left(-\left(p^{\alpha} \lambda\right)\right]=t^{\mu-1} \phi\left(-\alpha, \mu,-\lambda t^{-\alpha}\right)\right.
\end{gathered}
$$

(see (3.2.7) in $[20])$,

$$
D_{t}^{\nu}\left(t^{\mu-1} \phi\left(-\alpha, \mu,-\lambda t^{-\alpha}\right)\right)=t^{\mu-\nu-1} \phi\left(-\alpha, \mu-\nu,-\lambda t^{-\alpha}\right)
$$

(see (8) in [19]), and the convolution formula, we deduce (3.6) from (3.9). The lemma is proved.

$\mathrm{R}$ e $\mathrm{m}$ a $\mathrm{r} \mathrm{k}$. Let us recall the fundamental solution to the Cauchy problem for the heat equation

$$
\Gamma_{1}(x, t)=(4 \pi t)^{-n / 2} \exp \left(-\frac{|x|^{2}}{4 t}\right) .
$$

We can rewrite $\Gamma_{\alpha}$ in a more convenient form

$$
\Gamma_{\alpha}(x, t)=\int_{0}^{\infty} \Gamma_{1}(x, \lambda) t^{-1} \phi\left(-\alpha, 0,-\lambda t^{-\alpha}\right) d \lambda
$$


and use below the well-known estimate

$$
\left|D_{x}^{l} \Gamma_{1}(x, \lambda)\right| \leq c \lambda^{-\frac{n+|l|}{2}} \exp \left(-C \frac{|x|^{2}}{\lambda}\right)
$$

in analysis of $\Gamma_{\alpha}(x, t)$.

We assume that

$$
h_{n} \leq-\delta_{0}<0, \quad\left|h^{\prime}\right| \leq M_{0}
$$

Lemma 3.2. Let assumptions (3.4), (3.16) hold. Then the solutions of (3.1) and (3.2) are represented as

$$
\begin{gathered}
v(x, t)=-2\left(\frac{\partial \Gamma_{\alpha}}{\partial x_{n}} *_{2} \Phi\right) \equiv-2 \int_{-\infty}^{t} \int_{\mathbb{R}^{n}} \frac{\partial \Gamma_{\alpha}}{\partial x_{n}}\left(x^{\prime}-y^{\prime}, x_{n}, t-\tau\right) \Phi\left(y^{\prime}, \tau\right) d y^{\prime} \\
w(x, t)=\left(G *_{2} \Psi\right), \text { where } G(x, t)=-2 \int_{0}^{\infty} \frac{\partial \Gamma_{\alpha}}{\partial x_{n}}(x-h \lambda, t) d \lambda
\end{gathered}
$$

The proof of this Lemma is given in Appendix 5.1. In our analysis we follow very closely the classical approach of V.A. Solonnikov (see Chapter IV in [12]). First, we derive the estimates of $D_{t}^{\nu} D_{x}^{l} \Gamma_{\alpha}(x, t)(\nu \in \mathbb{R},|l| \geq 0)$. Then we establish some integral estimates of $\Gamma_{\alpha}, G$ and their derivatives. Finally, we estimate Hölder constants with respect to $t$ and $x$ of the potentials $\left(\Gamma_{\alpha} * f\right),\left(\Gamma_{\alpha} *_{1} \Phi\right)$ and $\left(G *_{2} \Phi\right)$.

Lemma 3.3. Let $\nu \in \mathbb{R},|l| \geq 0$. The function $\Gamma_{\alpha}(x, t)$, defined by (3.6), satisfies the following estimates $\left(\beta=\frac{\alpha}{2}\right)$ :

$$
\left|D_{t}^{\nu} D_{x}^{l} \Gamma_{\alpha}(x, t)\right| \leq c t^{\beta(2-n-|l|)-\nu-1} \gamma_{p(\nu, l)}\left(|x| t^{-\beta}\right) \exp \left(-\sigma\left(|x| t^{-\beta}\right)^{\frac{1}{1-\beta}}\right),
$$

here $(z>0)$,

$$
\gamma_{m}(z)=\left\{\begin{array}{l}
1, \text { if } m \leq 3, \\
|\log z|+1, \text { if } m=4, \quad p(\nu, l)=\left\{\begin{array}{l}
n+|l|, \text { if } \nu \in \mathbb{N} \cup\{0\}, \\
z^{4-n}, \text { if } m \geq 5,
\end{array} \quad \text { if }{ }^{4} \notin|l|+2, \text { if } \notin \mathbb{N} \cup\{0\} .\right.
\end{array}\right.
$$

The proof of this lemma is given in Appendix 5.2. Estimates (3.19) are established in [19] for the case $0 \leq|l| \leq 2$.

Estimates (3.19) allow us to deduce the integral estimates of $\Gamma_{\alpha}, G$.

Lemma 3.4. The following estimate holds:

$$
\int_{0}^{\infty}\left|D_{x}^{l} \Gamma_{\alpha}(x, t)\right| d t \leq C|x|^{-n+2-|l|}, \quad n+|l| \geq 3 .
$$


Lemma 3.5. The function $G$, defined by formula (3.18), satisfies the following estimates:

$$
\begin{gathered}
\int_{0}^{\infty}\left|D_{x}^{l} G(x, t)\right| d t \leq C|x|^{-n+2-|l|}, \quad 0 \leq|l| \leq 2, \\
\int_{0}^{\infty} t^{\beta / 2}\left|G_{x_{i}}(x, t)\right| d t \leq C|x|^{-n+3 / 2}, \quad i=1, \ldots, n, \\
\int_{0}^{\infty} t^{\delta \beta}\left|D_{t}^{\alpha} D_{x}^{l} G(x, t)\right| d t \leq C|x|^{\delta-n-|l|}, \quad|l|=0,1, \delta>0, \\
\int_{\mathbb{R}^{n-1}}\left|D_{t}^{\alpha-1+\zeta} G(x, t)\right| d x^{\prime} \leq C t^{-\beta-\zeta}, \quad \zeta \in \mathbb{R}, \\
\int_{\mathbb{R}^{n-1}}\left|D_{t}^{k} G_{x_{i}}(x, t)\right| d x^{\prime} \leq C t^{-1-k}, \quad k=0,1, \quad i=1, \ldots, n, \\
\int_{\mathbb{R}^{n-1}}^{l} G(x, t)\left|d x^{\prime} \leq C t^{\beta-1-\beta|l|}, \quad 0 \leq\right| l \mid \leq 2 .
\end{gathered}
$$

P r o o f of Lemma 3.4. By (3.19), we get

$$
\left|D_{x}^{l} \Gamma_{\alpha}(x, t)\right| \leq C \int_{0}^{\infty} t^{\beta(2-n-|l|)-1} \gamma_{n+|l|}\left(|x| t^{-\beta}\right) \exp \left(-\sigma\left(|x| t^{-\beta}\right)^{\frac{1}{1-\beta}}\right) d t .
$$

By the change of variable $t \rightarrow \lambda=|x| t^{-\beta}$, we obtain

$$
\left|D_{x}^{l} \Gamma_{\alpha}(x, t)\right| \leq c|x|^{-n+2-|l|} \int_{0}^{\infty} \lambda^{n+|l|-3} \gamma_{n+|l|}(\lambda) \exp \left(-\sigma \lambda^{\frac{1}{1-\beta}}\right) d \lambda \leq c|x|^{-n+2-|l|},
$$

where we used the definition of $\gamma_{m}$ and the assumption $n+|l| \geq 3$. The proof is finished.

$\mathrm{P}$ r o o f of Lemma 3.5. Begin with considering the expression $\sum_{i=1}^{n}\left(x_{i}-\right.$ $\left.h_{i} \lambda\right)^{2}$. We claim that

$$
\sum_{i=1}^{n}\left(x_{i}-h_{i} \lambda\right)^{2} \geq C_{0}^{2}\left(\sum_{i=1}^{n-1} x_{i}^{2}+\lambda^{2}\right)+x_{n}^{2}, \quad C_{0}^{2}=\frac{\delta_{0}^{2}}{2} \min \left\{1, \frac{1}{\delta_{0}^{2}+M_{0}^{2}}\right\} .
$$


It follows from (3.16) that for any $a \in(0,1)$,

$$
\begin{gathered}
\sum_{i=1}^{n}\left(x_{i}-h_{i} \lambda\right)^{2} \geq \sum_{i=1}^{n-1}\left(x_{i}^{2}-2 a x_{i} \frac{h_{i}}{a} \lambda+h_{i}^{2} \lambda^{2}\right)+x_{n}^{2}+h_{n}^{2} \lambda^{2} \\
\geq\left(1-a^{2}\right) \sum_{i=1}^{n-1} x_{i}^{2}-\left(\frac{1}{a^{2}}-1\right) M_{0}^{2} \lambda^{2}+\delta_{0}^{2} \lambda^{2}+x_{n}^{2} .
\end{gathered}
$$

Putting $a^{2}=\frac{1}{2}\left(1+\frac{M_{0}^{2}}{\delta_{0}^{2}+M_{0}^{2}}\right)$, we derive (3.27).

Let us establish $(3.22),(3.26)$. The rest of estimates can be proved by the same arguments.

Apply (3.18), (3.19) to see

$$
\begin{gathered}
J=\int_{0}^{\infty} t^{\beta / 2}\left|G_{x_{i}}(x, t)\right| d t \\
\leq C \int_{0}^{\infty} d t \int_{0}^{\infty} t^{\beta / 2-\beta n-1} \gamma_{n+2}\left(|x-h \lambda| t^{-\beta}\right) \exp \left(-\sigma\left(|x-d \lambda| t^{-\beta}\right)^{\frac{1}{1-\beta}}\right) d \lambda .
\end{gathered}
$$

Consider the cases $n=2$ and $n \geq 3$ separately.

If $n=2$, then from the inequality

$$
(|\log z|+1) \exp \left(-\sigma|z|^{\frac{1}{1-\beta}}\right) \leq C|z|^{-1 / 4} \exp \left(-\frac{\sigma}{2}|z|^{\frac{1}{1-\beta}}\right)
$$

and (3.27) it follows that

$$
\begin{gathered}
J \leq C \int_{0}^{\infty} d t \int_{0}^{\infty} t^{\beta / 2-2 \beta-1}\left(\frac{|x-h \lambda|}{t^{\beta}}\right)^{-1 / 4} \exp \left(-\frac{\sigma}{2}\left(\frac{|x-h \lambda|}{t^{\beta}}\right)^{\frac{1}{1-\beta}}\right) d \lambda \\
\leq C\left(C_{0}\right) \int_{0}^{\infty} d t \int_{0}^{\infty} t^{\beta / 2-2 \beta-1}\left(\frac{\left|x^{\prime}\right|}{t^{\beta}}\right)^{-\frac{1}{4}} \exp \left(-\frac{\sigma_{0}}{4}\left(\frac{\left|x^{\prime}\right|}{t^{\beta}}\right)^{\frac{1}{1-\beta}}\right) \exp \left(-\frac{\sigma_{0}}{4}\left(\frac{\lambda}{t^{\beta}}\right)^{\frac{1}{1-\beta}}\right) d \lambda,
\end{gathered}
$$

here and below $\sigma_{0}$ depends on $C_{0}$. By the change of variable $t \rightarrow s=\left|x^{\prime}\right| t^{-\beta}$, we deduce

$$
\begin{gathered}
J \leq C\left(C_{0}\right) \int_{0}^{\infty}\left(\frac{\left|x^{\prime}\right|}{s}\right)^{-\frac{1}{2}} s^{-\frac{1}{4}} \exp \left(-\frac{\sigma_{0}}{4} s^{\frac{1}{1-\beta}}\right) \frac{d s}{s} \\
\leq C\left(C_{0}\right)\left|x^{\prime}\right|^{-\frac{1}{2}} \int_{0}^{\infty} s^{-\frac{3}{4}} \exp \left(-\frac{\sigma_{0}}{4} s^{\frac{1}{1-\beta}}\right) d s \leq C\left(C_{0}\right)\left|x^{\prime}\right|^{-\frac{1}{2}} .
\end{gathered}
$$


Analogously, for $n \geq 3$, we get

$$
\begin{gathered}
J \leq C \int_{0}^{\infty} d t \int_{0}^{\infty} t^{\beta / 2-\beta n-1}\left(\frac{|x-h \lambda|}{t^{\beta}}\right)^{2-n} \exp \left(-\sigma\left(\frac{|x-h \lambda|}{t^{\beta}}\right)^{\frac{1}{1-\beta}}\right) d \lambda \\
\leq C\left(C_{0}\right) \int_{0}^{\infty} d t \int_{0}^{\infty} t^{\beta / 2-\beta n-1}\left(\frac{\left|x^{\prime}\right|}{t^{\beta}}\right)^{2-n} \exp \left(-\frac{\sigma_{0}}{2}\left(\frac{\left|x^{\prime}\right|}{t^{\beta}}\right)^{\frac{1}{1-\beta}}\right) \exp \left(-\frac{\sigma_{0}}{2}\left(\frac{\lambda}{t^{\beta}}\right)^{\frac{1}{1-\beta}}\right) d \lambda \\
\leq C\left(C_{0}\right) \int_{0}^{\infty} t^{\beta / 2-\beta(n-1)-1}\left(\frac{\left|x^{\prime}\right|}{t^{\beta}}\right)^{2-n} \exp \left(-\frac{\sigma_{0}}{2}\left(\frac{\left|x^{\prime}\right|}{t^{\beta}}\right)^{\frac{1}{1-\beta}}\right) d t \\
\leq C\left(C_{0}\right) \int_{0}^{\infty}\left(\frac{\left|x^{\prime}\right|}{s}\right)^{\frac{1}{2}-(n-1)} s^{2-n} \exp \left(-\frac{\sigma_{0}}{2} s^{\frac{1}{1-\beta}}\right) \frac{d s}{s} \\
\leq C\left(C_{0}\right)\left|x^{\prime}\right|^{\frac{3}{2}-n} \int^{\infty} s^{-\frac{1}{2}} \exp \left(-\frac{\sigma_{0}}{2} s^{\frac{1}{1-\beta}}\right) d s \leq C\left(C_{0}\right)\left|x^{\prime}\right|^{\frac{3}{2}-n} .
\end{gathered}
$$

Use this inequality and (3.29) to obtain (3.22).

By (3.18), (3.13), (3.14), we have

$$
\begin{gathered}
D_{x}^{l} G(x, t)=-2 \int_{0}^{\infty} D_{x}^{l} \frac{\partial \Gamma_{\alpha}}{\partial x_{n}}(x-h \mu, t) d \mu \\
=-2 \int_{0}^{\infty} d \lambda \int_{0}^{\infty} D_{x}^{l} \frac{\partial \Gamma_{1}}{\partial x_{n}}(x-h \mu, t) t^{-1} \phi\left(-\alpha, 0,-\lambda t^{-\alpha}\right) d \mu .
\end{gathered}
$$

Using (3.15), (2.8) and the change of variable $y^{\prime}=\frac{x^{\prime}-h \mu}{\lambda^{1 / 2}}$, we obtain

$$
\begin{gathered}
\mathcal{J}=\int_{\mathbb{R}^{n-1}}\left|D_{x}^{l} G(x, t)\right| d x^{\prime} \\
\leq C \int_{0}^{\infty} d \lambda \int_{0}^{\infty} \lambda^{-\frac{|l|}{2}-1} \exp \left(-C \frac{\left(x_{n}-h_{n} \mu\right)^{2}}{\lambda} t^{-1} \phi\left(-\alpha, 0,-\lambda t^{-\alpha}\right) d \mu .\right.
\end{gathered}
$$

The change of variable $\zeta=\frac{\mu}{\sqrt{\lambda}}$ and assumption (3.16) give

$$
\mathcal{J} \leq C \int_{0}^{\infty} \lambda^{-\frac{|l|+1}{2}} t^{-1} \phi\left(-\alpha, 0,-\lambda t^{-\alpha}\right) d \lambda \int_{0}^{\infty} \exp \left(-C \delta_{0} \zeta^{2}\right) d \zeta
$$


Estimate (2.9), formula (2.11) and the change of variable $\lambda \rightarrow \eta=\frac{\lambda}{t^{\beta}}$ imply

$$
\mathcal{J} \leq C t^{\beta-1-\beta|l|} \int_{0}^{\infty} \eta^{\frac{1-|l|}{2}} \exp \left(-\sigma \eta^{\frac{1}{1-\alpha}} d \eta \leq C t^{\beta-1-\beta|l|} \text { if } 0 \leq|l| \leq 2 .\right.
$$

The proof of (3.26) is finished.

Now we estimate Hölder constants of the potentials derived in (3.6), (3.17), (3.18).

Lemma 3.6. The following estimates hold:

$$
\begin{gathered}
\left\langle\frac{\partial^{2}\left(\Gamma_{\alpha} * f\right)}{\partial x_{i} \partial x_{j}}\right\rangle_{\alpha, \mathbb{R}_{T}^{n}}^{(\theta)} \leq C\langle f\rangle_{\alpha, \mathbb{R}_{T}^{n}}^{(\theta)} \\
\left\langle\frac{\partial\left(\Gamma_{\alpha} * f\right)}{\partial x_{i}}\right\rangle_{t, \mathbb{R}_{T}^{n}}^{\left(\frac{1+\theta}{2} \alpha\right)} \leq C\langle f\rangle_{\alpha, \mathbb{R}_{T}^{n},}^{(\theta)}, \\
\left\langle\left(D_{t}^{\alpha-1} \Gamma_{\alpha} *_{1} u_{0}\right)\right\rangle_{x, \mathbb{R}_{T}^{n}}^{(\theta)} \leq C\left\langle u_{0}\right\rangle_{x, \mathbb{R}^{n}}^{(\theta)}, \\
\left\langle\left(D_{t}^{\alpha-1} \Gamma_{\alpha} *_{1} u_{0}\right)\right\rangle_{t, \mathbb{R}_{T}^{n}}^{\left(\frac{k+\theta}{2} \alpha\right)} \leq C\left\langle u_{0}\right\rangle_{x, \mathbb{R}^{n}}^{(k+\theta)}, \quad k=0,1 .
\end{gathered}
$$

Lemma 3.7. The following estimates hold:

$$
\begin{gathered}
\left\langle\left(G_{x_{i}} *_{2} \psi\right)\right\rangle_{x, \mathbb{R}_{+, T}^{n}}^{(\theta)} \leq C\langle\psi\rangle_{\alpha, \mathbb{R}_{T}^{n-1}}^{(\theta)}, \\
\left\langle\partial_{t}^{\alpha}\left(G *_{2} \psi\right)\right\rangle_{t, \mathbb{R}_{+, T}^{n}}^{\left(\frac{\theta}{2} \alpha\right)} \leq C\langle\psi\rangle_{t, \mathbb{R}_{T}^{n-1}}^{\left(\frac{1+\theta}{2} \alpha\right)}, \\
\left\langle\partial_{t}^{\alpha}\left(G *_{2} \Psi\right)\right\rangle_{x, \mathbb{R}_{+, T}^{n}}^{(\theta)} \leq C\langle\psi\rangle_{t, \mathbb{R}_{T}^{n-1}}^{\left(\frac{1+\theta}{2} \alpha\right)}, \\
\left\langle\left(G_{x_{i} *_{2}} \psi\right)\right\rangle_{t, \mathbb{R}_{+, T}^{n}}^{(\theta \alpha)} \leq C\langle\psi\rangle_{\alpha, \mathbb{R}_{T}^{n-1}}^{(2 \theta)}, \\
\left\langle\left(G *_{2} \psi\right)\right\rangle_{t, \mathbb{R}_{+, T}^{n}}^{\left(\frac{1+\theta}{2} \alpha\right)} \leq C\langle\psi\rangle_{\alpha, \mathbb{R}_{T}^{n-1}}^{(\theta)}
\end{gathered}
$$

The proof of Lemma 3.6 follows the same line as the proof of $(2.1),(2.2)$ in $\S 2$, Chapter IV [12] and is based on estimates (3.19), (3.20). Thus it is omitted.

P r o o f of Lemma 3.7. Similarly to Chapter IV [12], we write the potential $\left(G_{x_{i}} *_{2} \psi\right)$ in the form

$$
\left(G_{x_{i}} *_{2} \psi\right)(x, t)=\int_{0}^{\infty} d \tau \int_{\mathbb{R}^{n-1}} G_{x_{i}}\left(x^{\prime}-y^{\prime}, x_{n}, \tau\right)\left(\psi\left(y^{\prime}, t-\tau\right)-\psi\left(y^{\prime}, t\right)\right) d y^{\prime}
$$




$$
\begin{gathered}
+\int_{0}^{\infty} d \tau \int_{\mathbb{R}^{n-1}} G_{x_{i}}\left(x^{\prime}-y^{\prime}, x_{n}, \tau\right)\left(\psi\left(y^{\prime}, t\right)-\psi\left(x^{\prime}, t\right)\right) d y^{\prime} \\
\quad+\psi\left(x^{\prime}, t\right) \int_{0}^{\infty} d \tau \int_{\mathbb{R}^{n-1}} G_{x_{i}}\left(x^{\prime}-y^{\prime}, x_{n}, \tau\right) d y^{\prime}
\end{gathered}
$$

Now we represent the difference $\left(G_{x_{i}} *_{2} \psi\right)(x, t)-\left(G_{x_{i}} *_{2} \psi\right)(\bar{x}, t)$ as the sum of seven integrals

$$
\begin{aligned}
& \left(G_{x_{i}} *_{2} \psi\right)(x, t)-\left(G_{x_{i} *_{2}} \psi\right)(\bar{x}, t) \\
& =\int_{0}^{|x-\bar{x}|^{1 / \beta}} d \tau \int_{\mathbb{R}^{n-1}} G_{x_{i}}\left(x^{\prime}-y^{\prime}, x_{n}, \tau\right)\left(\psi\left(y^{\prime}, t-\tau\right)-\psi\left(y^{\prime}, t\right)\right) d y^{\prime} \\
& -\int_{0}^{|x-\bar{x}|^{1 / \beta}} d \tau \int_{\mathbb{R}^{n-1}} G_{\bar{x}_{i}}\left(\bar{x}^{\prime}-y^{\prime}, \bar{x}_{n}, \tau\right)\left(\psi\left(y^{\prime}, t-\tau\right)-\psi\left(y^{\prime}, t\right)\right) d y^{\prime} \\
& +\int_{|x-\bar{x}|^{1 / \beta}}^{\infty} d \tau \int_{\mathbb{R}^{n-1}}\left(G_{x_{i}}\left(x^{\prime}-y^{\prime}, x_{n}, \tau\right)-G_{\bar{x}_{i}}\left(\bar{x}^{\prime}-y^{\prime}, \bar{x}_{n}, \tau\right)\left(\psi\left(y^{\prime}, t-\tau\right)-\psi\left(y^{\prime}, t\right)\right) d y^{\prime}\right. \\
& +\int_{K}\left(\psi\left(y^{\prime}, t\right)-\psi\left(x^{\prime}, t\right)\right) d y^{\prime} \int_{0}^{\infty} G_{x_{i}}\left(x^{\prime}-y^{\prime}, x_{n}, \tau\right) d \tau \\
& -\int_{K}\left(\psi\left(y^{\prime}, t\right)-\psi\left(\bar{x}^{\prime}, t\right)\right) d y^{\prime} \int_{0}^{\infty} G_{\bar{x}_{i}}\left(\bar{x}^{\prime}-y^{\prime}, \bar{x}_{n}, \tau\right) d \tau \\
& +\int_{\mathbb{R}^{n-1} \backslash K} d y^{\prime} \int_{0}^{\infty}\left(G_{x_{i}}\left(x^{\prime}-y^{\prime}, x_{n}, \tau\right)-G_{\bar{x}_{i}}\left(\bar{x}^{\prime}-y^{\prime}, \bar{x}_{n}, \tau\right)\right)\left(\psi\left(y^{\prime}, t\right)-\psi\left(\bar{x}^{\prime}, t\right)\right) d \tau \\
& -\left(\psi\left(\bar{x}^{\prime}, t\right)-\psi\left(x^{\prime}, t\right) \int_{0}^{\infty} d \tau \int_{K} G_{x_{i}}\left(x^{\prime}-y^{\prime}, x_{n}, \tau\right) d x^{\prime}=\sum_{i=1}^{7} I_{i},\right.
\end{aligned}
$$

here we use the notation

$$
K=\left\{y^{\prime} \in \mathbb{R}^{n-1}:\left|x^{\prime}-y^{\prime}\right| \leq 2|x-\bar{x}|\right\} .
$$


Applying (3.25) to $I_{1}, I_{2}$, we obtain

$$
\left|I_{1}\right|+\left|I_{2}\right| \leq c\langle\psi\rangle_{t, \mathbb{R}_{T}^{n-1}}^{\left(\frac{\theta \alpha}{2}\right)} \int_{0}^{|x-\bar{x}|^{1 / \beta}} \tau^{\frac{\theta \alpha}{2}-1} d \tau \leq c\langle\psi\rangle_{t, \mathbb{R}_{T}^{n-1}}^{\left(\frac{\theta \alpha}{2}\right)}|x-\bar{x}|^{\theta} .
$$

As for $I_{3}$, we represent it as

$$
\begin{gathered}
I_{3}=\sum_{k=1}^{n} \int_{|x-\bar{x}|^{1 / \beta}}^{\infty} d \tau \int_{0}^{1} d \lambda \int_{\mathbb{R}^{n-1}} G_{\eta_{i} \eta_{k}}\left(\eta^{\prime}-y^{\prime}, \eta_{n}, \tau\right)\left(x_{k}-\bar{x}_{k}\right) \\
\times\left(\psi\left(y^{\prime}, t-\tau\right)-\psi\left(y^{\prime}, t\right)\right) d y^{\prime}
\end{gathered}
$$

where $\eta=\bar{x}+\lambda(x-\bar{x})$. By (3.26), we get

$$
\left|I_{3}\right| \leq c\langle\psi\rangle_{t, \mathbb{R}_{T}^{n-1}}^{\left(\frac{\theta \alpha}{2}\right)}|x-\bar{x}| \int_{|x-\bar{x}|^{1 / \beta}}^{\infty} \tau^{\frac{\theta \alpha}{2}-1-\beta} d \tau \leq c\langle\psi\rangle_{t, \mathbb{R}_{T}^{n-1}}^{\left(\frac{\theta \alpha}{2}\right)}|x-\bar{x}|^{\theta} .
$$

With the help of (3.21) we derive

$$
\begin{gathered}
\left|I_{4}\right|+\left|I_{5}\right| \leq c\langle\psi\rangle_{x, \mathbb{R}_{T}^{n-1}}^{\theta}\left(\int_{K}\left|x^{\prime}-y^{\prime}\right|^{\theta-(n-1)} d y^{\prime}+\int_{\left|\bar{x}^{\prime}-y^{\prime}\right| \leq 3|\bar{x}-x|}\left|\bar{x}-y^{\prime}\right|^{\theta-(n-1)} d y^{\prime}\right) \\
\leq c\langle\psi\rangle_{x, \mathbb{R}_{T}^{n-1}}^{(\theta)}|x-\bar{x}|^{\theta}
\end{gathered}
$$

Now we have

$I_{6}=\sum_{k=1, n^{\prime}}^{n} \int_{\mathbb{R}^{n-1} \backslash K} d y^{\prime} \int_{0}^{1} d \lambda \int_{0}^{\infty} G_{\eta_{i} \eta_{k}}\left(\eta^{\prime}-y^{\prime}, \eta_{n}, \tau\right)\left(x_{k}-\bar{x}_{k}\right)\left(\psi\left(y^{\prime}, t\right)-\psi\left(\bar{x}^{\prime}, t\right)\right) d \tau$, where $\eta=\bar{x}+\lambda(x-\bar{x})$. One can easily check that

$$
|x-\bar{x}| \leq\left|\eta^{\prime}-y^{\prime}\right|, \quad\left|\bar{x}^{\prime}-y^{\prime}\right| \leq 2\left|\eta^{\prime}-y^{\prime}\right|
$$

for any $\eta=\bar{x}+\lambda(x-\bar{x}), y^{\prime} \in K, \lambda \in(0,1)$. From this fact and estimate (3.21) it follows that

$$
\left|I_{6}\right| \leq c\langle\psi\rangle_{x, \mathbb{R}_{T}^{n-1}}^{(\theta)}|x-\bar{x}| \int_{0}^{1} d \lambda \int_{\left|\eta^{\prime}-y^{\prime}\right| \geq|x-\bar{x}|}\left|\eta^{\prime}-y^{\prime}\right|^{\theta-n} d y^{\prime}
$$




$$
\leq c\langle\psi\rangle_{x, \mathbb{R}_{T}^{n-1}}^{(\theta)}|x-\bar{x}|^{\theta} .
$$

Then we decompose $I_{7}$ into the sum

$$
\begin{gathered}
I_{7}=\left(\psi\left(\bar{x}^{\prime}, t\right)-\psi\left(x^{\prime}, t\right)\right)\left(\int_{|x-\bar{x}|^{1 / \beta}}^{\infty} d \tau \int_{K} G_{x_{i}}\left(x^{\prime}-y^{\prime}, x_{n}, \tau\right) d y^{\prime}\right. \\
\left.+\int_{0}^{|x-\bar{x}|^{1 / \beta}} d \tau \int_{K} G_{x_{i}}\left(x^{\prime}-y^{\prime}, x_{n}, \tau\right) d y^{\prime}\right)=\left(\psi\left(\bar{x}^{\prime}, t\right)-\psi\left(x^{\prime}, t\right)\left(J^{\prime}+J^{\prime \prime}\right) .\right.
\end{gathered}
$$

By (3.22), we obtain

$$
\begin{gathered}
\left|J^{\prime}\right| \leq|x-\bar{x}|^{-1 / 2} \int_{|x-\bar{x}|^{1 / \beta}}^{\infty} d \tau \int_{K} \tau^{\beta / 2}\left|G_{x_{i}}\left(x^{\prime}-y^{\prime}, x_{n}, \tau\right)\right| d y^{\prime} \\
\leq c|x-\bar{x}|^{-1 / 2} \int_{K} \frac{d y^{\prime}}{\left|x^{\prime}-y^{\prime}\right|^{n-3 / 2}} \leq c .
\end{gathered}
$$

Before proceeding to prove

$$
J^{\prime \prime} \leq c
$$

we establish the identity

$$
\begin{gathered}
G(x, t)=-\frac{2}{h_{n}} \Gamma_{\alpha}(x, t)+\frac{2}{h_{n}} \sum_{i=1}^{n-1} \int_{0}^{\infty} h_{i} \frac{\partial \Gamma_{\alpha}}{\partial x_{i}}(x-h \lambda, t) d \lambda \\
=G_{1}(x, t)+G_{2}(x, t) .
\end{gathered}
$$

Since

$$
\frac{d}{d \lambda} \Gamma_{\alpha}(x-h \lambda, t)=-\sum_{i=1}^{n-1} h_{i} \frac{\partial \Gamma_{\alpha}}{\partial x_{i}}(x-h \lambda, t)-h_{n} \frac{\partial \Gamma_{\alpha}}{\partial x_{i}}(x-h \lambda, t),
$$

we obtain

$$
-\Gamma_{\alpha}(x, t)=-\sum_{i=1}^{n-1} \int_{0}^{\infty} h_{i} \frac{\partial \Gamma_{\alpha}}{\partial x_{i}}(x-h \lambda, t) d \lambda-h_{n} \int_{0}^{\infty} \frac{\partial \Gamma_{\alpha}}{\partial x_{n}}(x-h \lambda, t) d \lambda .
$$

Hence (3.48) is true. 
Now we consider the cases $a) i \neq n$ and $b) i=n$. For the case $a$ ), we get

$$
\begin{aligned}
J^{\prime \prime} & =\int_{0}^{|x-\bar{x}|^{1 / \beta}} d \tau \int_{\Sigma} G\left(x^{\prime}-y^{\prime}, x_{n}, \tau\right) \nu_{i} d S_{y^{\prime}} \\
& =\int_{0}^{|x-\bar{x}|^{1 / \beta}} d \tau \int_{\Sigma} G_{2}\left(x^{\prime}-y^{\prime}, x_{n}, \tau\right) \nu_{i} d S_{y^{\prime}},
\end{aligned}
$$

here $\Sigma=\left\{y^{\prime}:\left|x^{\prime}-y^{\prime}\right|=2|x-\bar{x}|\right\}$ and $\nu$ is the unit outward normal to $\Sigma$ in $\mathbb{R}^{n-1}$. By (3.19), we obtain

$$
\begin{gathered}
\left|J^{\prime \prime}\right| \leq \int_{0}^{|x-\bar{x}|^{1 / \beta}} d \tau \int_{\Sigma}\left|G_{2}\left(x^{\prime}-y^{\prime}, x_{n}, \tau\right)\right| d S_{y^{\prime}} \\
\leq c \int_{0}^{|x-\bar{x}|^{1 / \beta}} d \tau \int_{0}^{\infty} d \lambda \int_{\Sigma} \tau^{\beta(1-n)-1} \gamma_{n+2}(\xi) \exp \left(-\sigma \xi^{\frac{1}{1-\beta}}\right) d S_{y^{\prime}},
\end{gathered}
$$

where $\xi=\left|x^{\prime}-y^{\prime}-h \lambda\right| \tau^{-\beta}$.

If $n=2$, we use (3.28), (3.27) to get

$$
\begin{gathered}
\left|J^{\prime \prime}\right| \leq\left. c \int_{0}^{|x-\bar{x}|^{1 / \beta}} \frac{d \tau}{\tau} \int_{0}^{\infty} z^{-\frac{1}{4}} \exp \left(-\frac{\sigma_{0}}{4} z^{\frac{1}{1-\beta}}\right)\right|_{z=\frac{|x-\bar{x}|}{\tau^{\beta}}} \exp \left(-\frac{\sigma_{0}}{4}\left(\frac{\lambda}{\tau^{\beta}}\right)^{\frac{1}{1-\beta}}\right) \frac{d \lambda}{\tau^{\beta}} \\
\leq\left. c \int_{0}^{|x-\bar{x}|^{1 / \beta}} z^{-\frac{1}{4}} \exp \left(-\frac{\sigma_{0}}{4} z^{\frac{1}{1-\beta}}\right)\right|_{z=\frac{|x-\bar{x}|}{\tau^{\beta}}} \frac{d \tau}{\tau} .
\end{gathered}
$$

By the change of variable $z=|x-\bar{x}| \tau^{-\beta}$, we get

$$
\left|J^{\prime \prime}\right| \leq c \int_{1}^{\infty} z^{-5 / 4} \exp \left(-\frac{\sigma_{0}}{4} z^{\frac{1}{1-\beta}}\right) d z \leq c, \quad n=2, \quad i \neq n .
$$

In a similar way, we get (see (3.49)) for $n \geq 3$ and $i \neq n$,

$$
\left|J^{\prime \prime}\right| \leq c|x-\bar{x}|^{n-2} \int_{1}^{\infty}\left(\frac{z}{|x-\bar{x}|}\right)^{n-2} z^{2-n} \exp \left(-\frac{\sigma_{0}}{4} z^{\frac{1}{1-\beta}}\right) \frac{d z}{z} \leq c .
$$


Here, let us recall that meas $\Sigma=2 \frac{\pi^{\frac{n-1}{2}}}{\Gamma(n-1)}(2|x-\bar{x}|)^{n-2}$.

In the case $b)$, by (3.48), (3.18) we have

$$
\frac{\partial G}{\partial x_{n}}=-\frac{2}{h_{n}} \frac{\partial \Gamma_{\alpha}}{\partial x_{n}}(x, t)-\frac{1}{h_{n}} \sum_{i=1}^{n-1} h_{i} \frac{\partial G}{\partial x_{i}}(x, t) .
$$

The sum in the right-hand side of (3.52) is already estimated.

In remains to prove

$$
I^{\prime \prime}=\int_{0}^{|x-\bar{x}|^{1 / \beta}} d \tau \int_{K}\left|\frac{\partial \Gamma_{\alpha}}{\partial x_{n}}\left(x^{\prime}-y^{\prime}, x_{n}, \tau\right)\right| d y^{\prime} \leq c .
$$

By (3.14), we get

$$
-2 \frac{\partial \Gamma_{\alpha}}{\partial x_{n}}=\frac{1}{(4 \pi)^{n / 2}} \int_{0}^{\infty} \frac{x_{n}}{\lambda} \lambda^{-n / 2} \exp \left(-\frac{|x|^{2}}{4 \lambda}\right) t^{-1} \phi\left(-\alpha, 0,-\lambda t^{-\alpha}\right) d \lambda .
$$

In view of $(2.8)$, we have

$$
-2 \frac{\partial \Gamma_{\alpha}}{\partial x_{n}}(x, t) \geq 0
$$

We establish the identity

$$
I \equiv-2 \int_{0}^{\infty} d t \int_{\mathbb{R}^{n-1}} \frac{\partial \Gamma_{\alpha}}{\partial x_{n}}\left(x^{\prime}, x_{n}, t\right) d x^{\prime}=1 .
$$

Then (3.53) follows immediately from

$$
I^{\prime \prime} \leq c \int_{0}^{\infty} d \tau \int_{\mathbb{R}^{n-1}}\left|\frac{\partial \Gamma_{\alpha}}{\partial x_{n}}\left(x^{\prime}, x_{n}, \tau\right)\right| d x^{\prime}=\frac{c}{2} .
$$

First, let us recall the identity (see (10) in [19]):

$$
\int_{0}^{\infty} \phi(-\alpha, 1-\alpha,-z) d z=1
$$

We use (3.54) and (2.11) to rewrite the integral $I$ in the form

$$
I=\frac{1}{(4 \pi)^{n / 2}} \int_{0}^{\infty} \exp \left(-\frac{x_{n}^{2}}{4 \lambda}\right) \frac{x_{n} d \lambda}{\lambda^{3 / 2}} \int_{0}^{\infty} \phi\left(-\alpha, 1-\alpha,-\lambda t^{-\alpha}\right) \frac{\alpha \lambda d t}{t^{1+\alpha}} \int_{\mathbb{R}^{n-1}} \exp \left(-\frac{\left|x^{\prime}\right|^{2}}{4 \lambda}\right) \frac{d x^{\prime}}{\lambda^{\frac{n-1}{2}}} .
$$


Then we change the variables $x^{\prime} \rightarrow y^{\prime}=\frac{x^{\prime}}{\sqrt{\lambda}}, t \rightarrow \eta=\lambda t^{-\alpha}, \lambda \rightarrow \mu=\frac{x_{n}}{\sqrt{\lambda}}$ to get

$$
I=\frac{2}{(4 \pi)^{n / 2}} \int_{0}^{\infty} \exp \left(-\frac{\mu^{2}}{4 \lambda}\right) d \mu \int_{0}^{\infty} \phi(-\alpha, 1-\alpha,-\eta) d \eta \int_{\mathbb{R}^{n-1}} \exp \left(-\frac{\left|y^{\prime}\right|^{2}}{4 \lambda}\right) d y^{\prime} .
$$

We apply (3.56) and

$$
\int_{-\infty}^{+\infty} \exp \left(-\frac{z^{2}}{4}\right) d z=2 \sqrt{\pi}
$$

to obtain $(3.55)$.

Thus the proof of $(3.47)$ is finished.

Using (3.41), (3.42), (3.43), (3.44), (3.45), (3.46), we obtain (3.34).

In Appendix 5.3, we derive

$$
\begin{gathered}
\partial_{t}^{\alpha}\left(G *_{2} \psi\right)(x, t)=\int_{0}^{\infty} d \tau \int_{\mathbb{R}^{n-1}} D_{t}^{\alpha} G\left(x^{\prime}-y^{\prime}, x_{n}, \tau\right) \\
\times\left(\psi\left(y^{\prime}, t-\tau\right)-\psi\left(y^{\prime}, \tau\right)\right) d y^{\prime} .
\end{gathered}
$$

In order to prove (3.35), we estimate the following difference $(t>\bar{t})$ :

$$
\begin{gathered}
\partial_{t}^{\alpha}\left(G *_{2} \psi\right)(x, t)-\partial_{t}^{\alpha}\left(G *_{2} \psi\right)(x, \bar{t})= \\
\int_{2 \bar{t}-t}^{t} d \tau \int_{\mathbb{R}^{n-1}} D_{t}^{\alpha} G\left(x^{\prime}-y^{\prime}, x_{n}, t-\tau\right)\left(\psi\left(y^{\prime}, \tau\right)-\psi\left(y^{\prime}, t\right)\right) d y^{\prime} \\
-\int_{2 \bar{t}-t}^{t} d \tau \int_{\mathbb{R}^{n-1}} D_{\bar{t}}^{\alpha} G\left(x^{\prime}-y^{\prime}, x_{n}, \bar{t}-\tau\right)\left(\psi\left(y^{\prime}, \tau\right)-\psi\left(y^{\prime}, \bar{t}\right)\right) d y^{\prime} \\
+\int_{-\infty}^{2 \bar{t}-t} d \tau \int_{\mathbb{R}^{n-1}}\left(D_{t}^{\alpha} G\left(x^{\prime}-y^{\prime}, x_{n}, t-\tau\right)-D_{\bar{t}}^{\alpha} G\left(x^{\prime}-y^{\prime}, x_{n}, \bar{t}-\tau\right)\right) \\
\times\left(\psi\left(y^{\prime}, \tau\right)-\psi\left(y^{\prime}, \bar{t}\right)\right) d y^{\prime} \\
+\int_{-\infty}^{2 \bar{t}-t} d \tau \int_{\mathbb{R}^{n-1}}\left(\psi\left(y^{\prime}, \bar{t}\right)-\psi\left(y^{\prime}, t\right)\right) D_{t}^{\alpha} G\left(x^{\prime}-y^{\prime}, x_{n}, t-\tau\right) d y^{\prime}=\sum_{i=1}^{4} I_{i} .
\end{gathered}
$$


By (3.24), we obtain

$$
\begin{aligned}
& \left|I_{1}\right|+\left|I_{2}\right| \leq c\langle\psi\rangle_{t, \mathbb{R}_{T}^{n-1}}^{\left(\frac{1+\theta}{2} \alpha\right)}\left(\int_{2 \bar{t}-t}^{t}(t-\tau)^{\frac{1+\theta}{2} \alpha-\beta-1} d \tau\right. \\
& \left.+\int_{2 \bar{t}-t}^{t}(\bar{t}-\tau)^{\frac{1+\theta}{2} \alpha-\beta-1} d \tau\right) \leq c\langle\psi\rangle_{t, \mathbb{R}_{T}^{n-1}}^{\left(\frac{1+\theta}{2} \alpha\right)}|t-\bar{t}|^{\frac{\theta}{2} \alpha}
\end{aligned}
$$

and

$$
\begin{gathered}
\left|I_{3}\right| \leq c\langle\psi\rangle_{t, \mathbb{R}_{T}^{n-1}}^{\left(\frac{1+\theta}{2} \alpha\right)} \int_{-\infty}^{2 \bar{t}-t} d \tau \int_{\bar{t}}^{t} \int_{\mathbb{R}^{n-1}}^{t} D_{s}^{\alpha+1} G\left(x^{\prime}-y^{\prime}, x_{n}, s-\tau\right)(\bar{t}-\tau)^{\frac{1+\theta}{2}} \alpha d y^{\prime} \\
\leq c\langle\psi\rangle_{t, \mathbb{R}_{T}^{n-1}}^{\left(\frac{1+\theta}{2} \alpha\right)} \int_{-\infty}^{2 \bar{t}-t} d \tau \int_{\bar{t}}^{t}(s-\tau)^{\beta-2}(\bar{t}-\tau)^{\frac{1+\theta}{2} \alpha} d s \\
\leq c\langle\psi\rangle_{t, \mathbb{R}_{T}^{n-1}}^{\left(\frac{1+\theta}{2} \alpha\right)} \int_{\bar{t}}^{t} d s \int_{-\infty}^{2 \bar{t}-t}(\bar{t}-\tau)^{\frac{\theta}{2} \alpha-2} d \tau \leq c\langle\psi\rangle_{t, \mathbb{R}_{T}^{n-1}}^{\left(\frac{1+\theta}{2} \alpha\right)}|t-\bar{t}|^{\frac{\theta}{2} \alpha}
\end{gathered}
$$

To this end, we obtain from (3.24), integrating with respect to $\tau$,

$$
\begin{aligned}
\left|I_{4}\right|=\mid & \left|\int_{\mathbb{R}^{n-1}}\left(\psi\left(y^{\prime}, t\right)-\psi\left(y^{\prime}, \bar{t}\right)\right) D_{t}^{\alpha-1} G\left(x^{\prime}-y^{\prime}, x_{n}, 2(t-\bar{t})\right) d y^{\prime}\right| \\
& \leq c\langle\psi\rangle_{t, \mathbb{R}_{T}^{n-1}}^{\left(\frac{1+\theta}{2} \alpha\right)}|t-\bar{t}|^{\frac{1+\theta}{2} \alpha-\beta}=c\langle\psi\rangle_{t, \mathbb{R}_{T}^{n-1}}^{\left(\frac{1+\theta}{2} \alpha\right)}|t-\bar{t}|^{\frac{\theta}{2} \alpha} .
\end{aligned}
$$

Inequalities (3.58)-(3.60) yield estimate (3.35).

Estimates (3.36), (3.37), (3.38) are proved in a similar manner with the help of classical methods [12]. The proof of Lemma (3.5) is finished.

Arguing as in $\S 2$, Chapter IV [12], from the results of Lemmas 3.4, 3.5 we can deduce the assertions of the following existence lemmas.

Lemma 3.8. Assume that assumptions (3.3) hold. Then problem (1.8), (1.9) has a unique solution $u \in C_{\alpha}^{2+\theta}\left(\mathbb{R}_{T}^{n}\right)$, and

$$
\langle u\rangle_{\alpha, \mathbb{R}_{T}^{n}}^{2+\theta} \leq C\left(\langle f\rangle_{\alpha, \mathbb{R}_{T}^{n}}^{\theta}+\left\langle u_{0}\right\rangle_{\mathbb{R}^{n}}^{2+\theta}\right) .
$$


Lemma 3.9. Assume that assumptions (3.4) hold. Then problem (3.1) has a unique solution $v \in C_{\alpha, 0}^{2+\theta}\left(\mathbb{R}_{+, T}^{n}\right)$, and

$$
\langle v\rangle_{\alpha, \mathbb{R}_{T}^{n}}^{2+\theta} \leq C\langle\Phi\rangle_{\alpha, \mathbb{R}_{T}^{n-1}}^{2+\theta}
$$

Lemma 3.10. Assume that assumptions (3.4), (3.16) hold. Then problem (3.2) has a unique solution $w \in C_{\alpha, 0}^{2+\theta}\left(\mathbb{R}_{+, T}^{n}\right)$, and

$$
\langle w\rangle_{\alpha, \mathbb{R}_{T}^{n}}^{2+\theta} \leq C\left(\delta_{0}, M_{0}\right)\langle\Psi\rangle_{\alpha, \mathbb{R}_{T}^{n-1}}^{1+\theta}
$$

\section{The Proof of Theorem 2.1}

First we consider the case of zero initial data:

Theorem 4.1. Let assumptions (1.6), (1.7), (2.2) hold. Suppose

$$
u_{0}=0, f \in C_{\alpha, 0}^{\theta}\left(\Omega_{T}\right), \psi_{1} \in C_{\alpha, 0}^{2+\theta}\left(\Sigma_{T}^{1}\right), \psi_{2} \in C_{\alpha, 0}^{1+\theta}\left(\Sigma_{T}^{2}\right) .
$$

Then problem (1.2)-(1.4) for sufficiently small $\tau \in(0, T)$ has a unique solution $u \in C_{\alpha, 0}^{2+\theta}\left(\Omega_{\tau}\right)$ satisfying the estimate

$$
|u|_{\alpha, \Omega_{\tau}}^{(2+\theta)} \leq c\left(|f|_{\alpha, \Omega_{\tau}}^{(\theta)}+\left|\psi_{1}\right|_{\alpha, \Sigma_{\tau}^{1}}^{(2+\theta)}+\left|\psi_{2}\right|_{\alpha, \Sigma_{\tau}^{2}}^{(1+\theta)}\right) .
$$

We prove this theorem by constructing a regularizer. Since it is a standard procedure (see Chapter IV [12]), we give only a sketch of the proof.

We cover the domain $\Omega$ with the balls $K_{\lambda}^{(k)}$ and $K_{2 \lambda}^{(k)}$ of radii $\lambda$ and $2 \lambda$, respectively, with a common center $\xi^{(k)}$ for sufficiently small $\lambda>0$.

The index $k$ belongs to one of the sets: $k \in \mathrm{N}^{(i)}$ if $K_{\lambda}^{(k)} \cap \Sigma^{i} \neq \emptyset, i=1,2$ and $k \in \mathrm{M}$ if $K_{\lambda}^{(k)} \cap \partial \Omega=\emptyset$.

We take

$$
\tau=\varkappa \lambda^{\frac{2}{\alpha}}, \quad \text { where } \varkappa<1 .
$$

Let $\zeta^{(k)}, \eta^{(k)}$ be the sets of smooth functions subordinated to the indicated overlapping of $\Omega$ such that

$$
\begin{gathered}
\sum_{k} \zeta^{(k)}(x) \eta^{(k)}(x)=1, \quad x \in \Omega, \\
\left|D_{x}^{l} \zeta^{(k)}\right|+\left|D_{x}^{l} \eta^{(k)}\right| \leq c \lambda^{-|l|},|l| \geq 0 .
\end{gathered}
$$

We suppose that the boundary $\Sigma^{i} \cap K_{2 \lambda}^{(k)}(i=1,2)$ can be given by the equation

$$
y_{n}=F\left(y^{\prime}\right)
$$


in some local coordinates $\{y\}$ with origin at the point $\xi^{(k)}$, where the axis $y_{n}$ is oriented at the direction of the outward normal vector $n\left(\xi^{(k)}\right)$ to the surface $\Sigma^{i}$.

We "straighten" the boundary by the formulae

$$
z^{\prime}=y^{\prime}, \quad z_{n}=y_{n}-F\left(y^{\prime}\right)
$$

Let $z=Z_{k}(x)$ be the transformation of the coordinates $\{x\}$ into $\{z\}$.

We denote

$$
\begin{gathered}
\mathcal{L}_{0}\left(x, t, \frac{\partial}{\partial x}, \partial_{t}^{\alpha}\right)=\partial_{t}^{\alpha}-\sum_{i, j=1}^{n} a_{i j}(x, t) \frac{\partial^{2}}{\partial x_{i} \partial x_{j}}, \\
\mathcal{B}_{0}\left(x, t, \frac{\partial}{\partial x}\right)=\sum_{i}^{n} b_{i}(x, t) \frac{\partial}{\partial x_{i}}
\end{gathered}
$$

Let $\mathcal{L}_{0}^{(k)}, \mathcal{B}_{0}^{(k)}$ be the operators $\mathcal{L}_{0}, \mathcal{B}_{0}$ in local coordinates $\{y\}$ at the point $\left(\xi^{(k)}, 0\right)$ :

$$
\begin{gathered}
\mathcal{L}_{0}^{(k)}\left(\xi^{(k)}, 0, \frac{\partial}{\partial y}, \partial_{t}^{\alpha}\right)=\partial_{t}^{\alpha}-\sum_{i, j=1}^{n} a_{i j}^{(k)} \frac{\partial^{2}}{\partial y_{i} \partial y_{j}}, \\
\mathcal{B}_{0}^{(k)}\left(\xi^{(k)}, 0, \frac{\partial}{\partial y}\right)=\sum_{i}^{n} b_{i}^{(k)} \frac{\partial}{\partial y_{i}} .
\end{gathered}
$$

We set $g=\left(f, \psi_{1}, \psi_{2}\right)$ and define a regularizer $\mathcal{R}$ by the formulae

$$
\mathcal{R} g=\sum_{k} \eta^{(k)}(x) u_{k}(x, t)
$$

where the functions $u_{k}$ are found as follows.

If $k \in \mathrm{M}$, we find the function $u_{k}(x, t)$ as the solution to the Cauchy problem

$$
\begin{gathered}
\mathcal{L}_{0}^{(k)}\left(\xi^{(k)}, 0, \frac{\partial}{\partial y}, \partial_{t}^{\alpha}\right) u_{k}(x, t)=f_{k}(x, t), \quad(x, t) \in \mathbb{R}_{T}^{n}, \\
u_{k}(x, 0)=0, \quad x \in \mathbb{R}^{n},
\end{gathered}
$$

here $f_{k}(x, t)=\zeta_{k}(x) f(x, t)$.

If $k \in \mathrm{N}^{(1)} \cup \mathrm{N}^{(2)}$, we set

$$
f_{k}^{\prime}(z, t)=\left.\zeta_{k}(x) f(x, t)\right|_{x=Z_{k}(z)}, \psi_{i, k}^{\prime}(z, t)=\left.\zeta_{k}(x) \psi_{i}(x, t)\right|_{x=Z_{k}(z)}, \quad i=1,2 .
$$

If $k \in \mathrm{N}^{(1)}$, we find the function $u_{k}^{\prime}(x, t)$ as the solution to the first boundary value problem

$$
\mathcal{L}_{0}^{(k)}\left(\xi^{(k)}, 0, \frac{\partial}{\partial z}, \partial_{t}^{\alpha}\right) u_{k}^{\prime}(z, t)=f_{k}^{\prime}(z, t), \quad(x, t) \in \mathbb{R}_{+, T}^{n},
$$




$$
u_{k}^{\prime}\left(z^{\prime}, 0, t\right)=\psi_{1, k}^{\prime}\left(z^{\prime}, t\right), \quad\left(z^{\prime}, t\right) \in \mathbb{R}_{T}^{n-1}, \quad u_{k}^{\prime}(z, 0)=0, \quad z \in \mathbb{R}_{+}^{n} .
$$

If $k \in \mathrm{N}^{(2)}$, we find the function $u_{k}^{\prime}(x, t)$ as the solution to the oblique boundary value problem

$$
\begin{gathered}
\mathcal{L}_{0}^{(k)}\left(\xi^{(k)}, 0, \frac{\partial}{\partial z}, \partial_{t}^{\alpha}\right) u_{k}^{\prime}(z, t)=f_{k}^{\prime}(z, t), \quad(x, t) \in \mathbb{R}_{+, T}^{n} \\
\mathcal{B}_{0}^{(k)}\left(\xi^{(k)}, 0, \frac{\partial}{\partial z}\right) u_{k}^{\prime}\left(z^{\prime}, 0, t\right)=\psi_{2, k}^{\prime}\left(z^{\prime}, t\right),\left(z^{\prime}, t\right) \in \mathbb{R}_{T}^{n-1} \\
u_{k}^{\prime}(z, 0)=0, z \in \mathbb{R}_{+}^{n} .
\end{gathered}
$$

Now we set

$$
u_{k}(x, t)=\left.u_{k}^{\prime}(z, t)\right|_{z=Z_{k}^{-1}(x)}, \quad k \in \mathrm{N}^{(1)} \cup \mathrm{N}^{(2)} .
$$

So we define $u_{k}(x, t)$ for all $k$.

Due to $\S 6$, Chapter IV in [12], we can reduce problems (4.4), (4.5), (4.6) to the case $a_{i j}^{(k)}=\delta_{i j}$. Moreover, we can repeat routine calculations of $\S 6,7$ Chapter IV in [12] in order to prove that the parameter $\delta_{0}$ in (3.16) depends only on $\delta$ and $\mu$ from (1.6), (1.7).

For the sake of convenience, problem (1.2)-(1.4) (with zero initial data) can be conventionally written in the operator form

$$
\mathcal{A} u=g,
$$

where $\mathcal{A} u$ is a linear operator determined by the expressions in the left-hand sides of (1.2), (1.4). Moreover, $\mathcal{A}: C_{\alpha, 0}^{2+\theta}\left(\Omega_{T}\right) \rightarrow \mathcal{H}\left(\Omega_{T}\right)$, where $\mathcal{H}\left(\Omega_{T}\right)=C_{\alpha, 0}^{\theta}\left(\Omega_{T}\right) \times$ $C_{\alpha, 0}^{2+\theta}\left(\Sigma_{T}^{1}\right) \times C_{\alpha, 0}^{1+\theta}\left(\Sigma_{T}^{2}\right)$ represents the space of functions $g=\left(f, \psi_{1}, \psi_{2}\right)$ with the norm

$$
|g|_{\mathcal{H}\left(\Omega_{T}\right)}=|f|_{\alpha, \Omega_{T}}^{(\theta)}+\left|\psi_{1}\right|_{\alpha, \Sigma_{T}^{1}}^{(2+\theta)}+\left|\psi_{2}\right|_{\alpha, \Sigma_{T}^{2}}^{(1+\theta)}
$$

On the base of estimates (3.61)-(3.63), we obtain

$$
|\mathcal{R} g|_{\alpha, \Omega_{\tau}}^{(2+\theta)} \leq c|g|_{\mathcal{H}\left(\Omega_{\tau}\right)},
$$

where $c$ does not depend on $\lambda$ and $\tau$, and for any $h \in \mathcal{H}\left(\Omega_{\tau}\right), u \in C_{\alpha}^{(2+\theta)}\left(\Omega_{T}\right)$,

$$
\begin{aligned}
|\mathcal{R} \mathcal{A} u-u|_{\left.\alpha, \Omega_{T}\right)}^{2+\theta} & \leq \frac{1}{2}|u|_{\left.\alpha, \Omega_{T}\right)}^{(2+\theta)}, \\
|\mathcal{A R} g-g|_{\mathcal{H}\left(\Omega_{T}\right)} & \leq \frac{1}{2}|g|_{\mathcal{H}\left(\Omega_{\tau}\right)}
\end{aligned}
$$

if $\tau$ is sufficiently small and (4.3) is in force. The calculations are simple but tedious. Inequalities (4.7), (4.8) yield the assertion of Theorem 4.1. It should 
be emphasized that in our analysis we follow very closely the standard technique found in Chapter IV [12].

To remove the restrictions imposed on the initial data in (4.1) and then extend the solution from $[0, \tau]$ to $[0, T]$, we reduce problem $(1.2)-(1.4)$ to the new unknowns with zero initial data $a$ ) at $t=0, b)$ at $t=\tau$.

In the case $a$ ), we set

$$
\begin{gathered}
u_{1}(x)=f(x, 0)+\sum_{i, j=1}^{n} a_{i j}(x, 0) \frac{\partial^{2} u_{0}(x)}{\partial x_{i} \partial x_{j}}+\sum_{i=1}^{n} a_{i}(x, 0) \frac{\partial u_{0}(x)}{\partial x_{i}}+a_{0}(x, 0) u_{0}(x), \\
f_{1}(x)=u_{1}(x)-\Delta u_{0}(x), \quad x \in \Omega .
\end{gathered}
$$

We observe that $u_{1}, f_{1} \in C^{\theta}(\Omega)$. By Hesten's lemma, we construct $\widehat{u}_{0} \in$ $C^{2+\theta}\left(\mathbb{R}^{n}\right), \widehat{f}_{1} \in C^{\theta}\left(\mathbb{R}_{T}^{n}\right)$ such that

$$
\widehat{u}_{0}(x)=u_{0}(x), \quad \widehat{f}_{1}(x)=f(x), \quad x \in \Omega .
$$

Then we determine the auxiliary function $u^{(0)}(x, t)$ under conditions

$$
u^{(0)}(x, 0)=u_{0}(x), \quad \partial_{t}^{\alpha} u^{(0)}(x, 0)=u_{1}(x), \quad x \in \Omega,
$$

as a solution to the Cauchy problem

$$
\begin{gathered}
\partial_{t}^{\alpha} u^{(0)}(x, t)-\Delta u^{(0)}(x, t)=\widehat{f}(x), \quad(x, t) \in \mathbb{R}_{T}^{n}, \\
u^{(0)}(x, 0)=\widehat{u}_{0}(x), \quad x \in \mathbb{R}^{n} .
\end{gathered}
$$

We employ Lemma 3.8 to deduce $u^{(0)} \in C_{\alpha}^{2+\theta}\left(\mathbb{R}_{T}^{n}\right)$ and

$$
\left|u^{(0)}\right|_{\alpha, \mathbb{R}_{T}^{n}}^{(2+\theta)} \leq c\left(\left|u_{0}\right|_{\mathbb{R}^{n}}^{(2+\theta)}+|f(\cdot, 0)|_{\mathbb{R}^{n}}^{(\theta)}\right) .
$$

Now we look for the solution of $(1.2)-(1.4)$ as $u(x, t)=v(x, t)+u^{(0)}(x, t)$, where $v$ is found from the problem of the form (1.2)-(1.4) with zero initial data and recalculated right-hand terms.

In the case $b)$, we have to find the function $u^{(\tau)}(x, t) \in C_{\alpha}^{2+\theta}\left(\Omega_{2 \tau}\right)$ satisfying the condition

$$
u^{(\tau)}(x, t)=u(x, t), \quad x \in \Omega, t \in[0, \tau],
$$

where $u(x, t)$ is a local solution of problem $(1.2)-(1.4)$ on $[0, \tau]$. We remark that (4.11) implies

$$
\partial_{t}^{\alpha} u^{(\tau)}(x, t)=\partial_{t}^{\alpha} u(x, t), \quad x \in \Omega, t \in[0, \tau] .
$$

It should be noticed that we need to know the function $u^{(\tau)}(x, t)$ on the whole segment $[0, \tau]$. This is connected with nonlocal property of the fractional derivative $\partial_{t}^{\alpha}$ 
By Hesten's lemma, again we can continue $u(x, t)$ to the set $\mathbb{R}_{\tau}^{n}$ :

$$
\widehat{u} \in C_{\alpha}^{2+\theta}\left(\mathbb{R}_{\tau}^{n}\right), \quad|\widehat{u}|_{\alpha, \mathbb{R}_{\tau}^{n}}^{(2+\theta)} \leq c|u|_{\alpha, \Omega_{\tau}}^{(2+\theta)}, \quad \widehat{u}(x, t)=u(x, t), \quad(x, t) \in \Omega_{\tau} .
$$

Then we put

$$
\omega(x, t)= \begin{cases}\partial_{t}^{\alpha} \widehat{u}(x, t)-\widehat{u}(x, t), & x \in \mathbb{R}^{n}, t \in[0, \tau], \\ \partial_{t}^{\alpha} \widehat{u}(x, \tau)-\widehat{u}(x, \tau), & x \in \mathbb{R}^{n}, t \in[\tau, 2 \tau] .\end{cases}
$$

Obviously, $\omega \in C_{\alpha}^{\theta}\left(\mathbb{R}_{2 \tau}^{n}\right)$. We are now in position to determine $u^{(\tau)}$ as a solution of the Cauchy problem

$$
\begin{gathered}
\partial_{t}^{\alpha} u^{(\tau)}(x, t)-\Delta u^{(\tau)}(x, t)=\omega(x, t), \quad(x, t) \in \mathbb{R}_{2 \tau}^{n}, \\
u^{(\tau)}(x, 0)=\widehat{u}_{0}(x), \quad x \in \mathbb{R}^{n} .
\end{gathered}
$$

After that we look for the solution of problem (1.2)-(1.4) in the form

$$
u(x, t)=v(x, t)+u^{(\tau)}(x, t),
$$

where $v(x, t)$ satisfies $(1.2),(1.4)$, and

$$
v(x, t)=0, \quad x \in \Omega, t \in[0, \tau] .
$$

The boundary value problem of the form (1.2)-(1.4) for $v$ is obtained by translation in time: $t \rightarrow t-\tau$ (see also [11]).

We can repeat this procedure in order to prove the solvability of problem (1.2)-(1.4) on segment $[0, T]$.

\section{Appendix}

\subsection{The proof of Lemma 3.2}

We take the Fourier transform $F^{\prime}$ in the tangent space variables $x^{\prime}$

$$
F^{\prime}[v]=\int_{\mathbb{R}^{n-1}} v(x, t) \exp \left(-i x^{\prime} \cdot \xi\right) d x^{\prime}, \quad \xi=\left(\xi_{1}, \ldots, \xi_{n-1}\right)
$$

and the Laplace transform in $t$ (3.7). Problem (3.1) then reduces to the ordinary differential equation $(\widetilde{v}=F[L[v]])$

$$
p^{\alpha} \widetilde{v}\left(\xi, x_{n}, p\right)+|\xi|^{2} \widetilde{v}\left(\xi, x_{n}, p\right)-\widetilde{v}_{x_{n} x_{n}}\left(\xi, x_{n}, p\right)=0
$$

with the boundary conditions

$$
\widetilde{v} \rightarrow 0, \quad x_{n} \rightarrow \infty, \quad \widetilde{v}(\xi, 0, p)=\widetilde{\Phi}(\xi, p) .
$$


We solve problem (5.1), (5.2) to obtain

$$
\widetilde{v}\left(\xi, x_{n}, p\right)=\exp \left(-\sqrt{p^{\alpha}+|\xi|^{2}} x_{n}\right) \widetilde{\Phi}(\xi, p) .
$$

We need to prove that

$$
L F^{\prime}\left[-2 \frac{\partial \Gamma_{\alpha}}{\partial x_{n}}\right]=\exp \left(-\sqrt{p^{\alpha}+|\xi|^{2}} x_{n}\right) .
$$

Then identity (3.17) follows from (5.4) and the convolution formula.

Since

$$
F^{\prime}\left[\exp \left(-\frac{\left|x^{\prime}\right|^{2}}{4 \lambda}\right]=\exp \left(-|\xi|^{2} \lambda\right)(4 \sqrt{\pi} \lambda)^{\frac{n-1}{2}}\right.
$$

and

$$
L\left[t^{-1} \phi\left(-\alpha, 0,-\lambda t^{-\alpha}\right)\right]=\exp \left(-p^{\alpha} \lambda\right)
$$

(see $(3.10),(3.11))$, we obtain from $(3.54)$

$$
L F^{\prime}\left[-2 \frac{\partial \Gamma_{\alpha}}{\partial x_{n}}\right]=\frac{1}{2 \sqrt{\pi}} \int_{0}^{\infty} x_{n} \lambda^{-3 / 2} \exp \left(-\frac{x_{n}^{2}}{4 \lambda}-\left(p^{\alpha}+|\xi|^{2}\right) \lambda\right) d \lambda .
$$

The change of variable $\lambda \rightarrow z=\frac{x_{n}}{\sqrt{\lambda}}$ and formula (3.325) in [5] give

$$
L F^{\prime}\left[-2 \frac{\partial \Gamma_{\alpha}}{\partial x_{n}}\right]=\frac{1}{\sqrt{\pi}} \int_{0}^{\infty} \exp \left(-\frac{z^{2}}{4}-\frac{\left(p^{\alpha}+|\xi|^{2}\right) x_{n}^{2}}{z^{2}}\right) d \lambda=\exp \left(-\sqrt{\left(p^{\alpha}+|\xi|^{2}\right) x_{n}^{2}}\right)
$$

Thus identity (5.4) is proved.

Then we apply the Laplace transform in $t$ and the Fourier transform in $x^{\prime}$ to problem (3.2) to obtain

$$
\begin{gathered}
p^{\alpha} \widetilde{w}\left(\xi, x_{n}, p\right)+|\xi|^{2} \widetilde{w}\left(\xi, x_{n}, p\right)-\widetilde{w}_{x_{n} x_{n}}\left(\xi, x_{n}, p\right)=0, \\
\left.\left(h_{n} \widetilde{w}_{x_{n}}+i h^{\prime} \cdot \xi \widetilde{w}\right)\right|_{x_{n}=0}=\widetilde{\Psi}(\xi, p), \quad \widetilde{w} \rightarrow 0, \quad x_{n} \rightarrow \infty .
\end{gathered}
$$

Assumption (3.16) allows us to write

$$
\begin{gathered}
\widetilde{w}\left(\xi, x_{n}, p\right)=\frac{\exp \left(-\sqrt{p^{\alpha}+|\xi|^{2}} x_{n}\right)}{-h_{n} \sqrt{p^{\alpha}+|\xi|^{2}}+i h^{\prime} \cdot \xi} \widetilde{\Psi}(\xi, p) \\
=\int_{0}^{\infty} \exp \left(-\sqrt{p^{\alpha}+|\xi|^{2}}\left(x_{n}-h_{n} \lambda\right)-i h^{\prime} \cdot \xi \lambda\right) d \lambda \widetilde{\Psi}(\xi, p) \equiv \widetilde{G}\left(\xi, x_{n}, p\right) \widetilde{\Psi}(\xi, p) .
\end{gathered}
$$

Identity (5.4) implies $L^{-1} F^{\prime-1}[\widetilde{G}]=-2 \int_{0}^{\infty} \frac{\partial \Gamma_{\alpha}}{\partial x_{n}}(x-h \lambda, t) d \lambda=G(x, t)$. 
To this end, we need to prove that potentials (3.17), (3.18) satisfy the boundary conditions in (3.1), (3.2).

As for (3.1), similarly to the classical case, we need the identity

$$
-2 \int_{0}^{\infty} d t \int_{\mathbb{R}^{n-1}} \frac{\partial \Gamma_{\alpha}}{\partial x_{n}}\left(x^{\prime}, x_{n}, t\right) d x^{\prime}=1
$$

derived above in Lemma 3.7 (see (3.55)).

As for problem (3.3), we see

$$
\begin{gathered}
\sum_{i=1}^{n} h_{i} \frac{\partial G}{\partial x_{i}}(x, t)=-2 \int_{0}^{\infty} \sum_{i=1}^{n} \frac{\partial^{2} \Gamma_{\alpha}}{\partial x_{n} \partial x_{i}}(x-h \lambda, t) d \lambda \\
=2 \int_{0}^{\infty} \frac{d}{d \lambda} \frac{\partial \Gamma_{\alpha}}{\partial x_{n}}(x-h \lambda, t) d \lambda=-2 \frac{\partial \Gamma_{\alpha}}{\partial x_{n}}(x, t) .
\end{gathered}
$$

This fact implies that $\left.\sum_{i=1}^{n} h_{i} \frac{\partial}{\partial x_{i}}\left(G *_{2} \Psi\right)\right|_{x_{n}=0}=\Psi$.

\subsection{The proof of Lemma 3.3}

By (3.14), (3.12), we obtain

$$
D_{x}^{l} D_{t}^{\nu} \Gamma_{\alpha}(x, t)=\int_{0}^{\infty} D_{x}^{l} \Gamma_{1}(x, \lambda) t^{-\nu-1} \phi\left(-\alpha,-\nu,-\lambda t^{-\alpha}\right) d \lambda .
$$

We prove (3.19) for $\nu \in \mathbb{N} \cup 0$. If $\nu \notin \mathbb{N} \cup 0$, the proof is the same. Inequalities (3.15) and (2.9) give

$$
\left|D_{x}^{l} D_{t}^{\nu} \Gamma_{\alpha}(x, t)\right| \leq c \int_{0}^{\infty} \lambda^{-\frac{n+|l|}{2}} \exp \left(-C \frac{|x|^{2}}{4 \lambda}\right) \lambda t^{-\alpha-\nu-1} \exp \left(-\sigma\left(\lambda t^{-\alpha}\right)^{\frac{1}{1-\alpha}}\right) d \lambda .
$$

By the change of variable $\lambda \rightarrow \eta=\lambda t^{-\alpha}$, we get

$$
\left|D_{x}^{l} D_{t}^{\nu} \Gamma_{\alpha}(x, t)\right| \leq c t^{\beta(2-n-|l|)-\nu-1} \int_{0}^{\infty} \eta^{1-\frac{n+|l|}{2}} \exp \left(-A\left(\frac{|x|^{2}}{t^{\alpha}} \frac{1}{\eta}+\eta^{\frac{1}{1-\alpha}}\right)\right) d \eta .
$$

It is not hard to prove $(z \geq 0)$

$$
\frac{z}{\eta}+\eta^{\frac{1}{1-\alpha}} \geq \sigma_{*}(\alpha) z^{\frac{1}{2-\alpha}} \text { for all } \eta \in(0, \infty) .
$$


Then it follows that

$$
\begin{gathered}
\left|D_{x}^{l} D_{t}^{\nu} \Gamma_{\alpha}(x, t)\right| \leq c t^{\beta(2-n-|l|)-\nu-1} \exp \left(-\sigma_{*} \frac{A}{2}\left(\frac{|x|}{t^{\beta}}\right)^{\frac{1}{1-\beta}}\right) \\
\quad \times \int_{0}^{\infty} \eta^{1-\frac{n+|l|}{2}} \exp \left(-\frac{A}{2}\left(\frac{|x|^{2}}{t^{\alpha}} \frac{1}{\eta}+\eta^{\frac{1}{1-\alpha}}\right)\right) d \eta .
\end{gathered}
$$

We set $(B>0, z>0)$,

$$
J_{m}(z)=\int_{0}^{\infty} \eta^{1-\frac{m}{2}} \exp \left(-B\left(\frac{z}{\eta}+\eta^{\frac{1}{1-\alpha}}\right)\right) d \eta
$$

and claim that

$$
J_{m}(z) \leq c\left\{\begin{array}{l}
1, \quad m=1,2,3 ; \\
|\log z|+1, \quad m=4 ; \\
z^{2-\frac{m}{2}}, \quad m \geq 5 .
\end{array}\right.
$$

If $m=1,2,3$, one can easily see

$$
J_{m}(z) \leq c \int_{0}^{\infty} \eta^{1-\frac{m}{2}} \exp \left(-B \eta^{\frac{1}{1-\alpha}}\right) d \eta \leq c .
$$

If $m=4$, we get

$$
\begin{gathered}
J_{4,1}(z)=\int_{0}^{z} \exp \left(-B \frac{z}{\eta}\right) \frac{d \eta}{\eta}=\int_{1}^{\infty} \exp (-B s) \frac{d s}{s} \leq c \\
J_{4,2}(z)=\int_{z}^{\infty} \exp \left(-B \eta^{\frac{1}{1-\alpha}}\right) \frac{d \eta}{\eta}=\left.\log \eta \exp \left(-B \eta^{\frac{1}{1-\alpha}}\right)\right|_{z} ^{\infty} \\
+\frac{B}{1-\alpha} \int_{z}^{\infty} \log \eta \eta^{\frac{\alpha}{1-\alpha}} \exp \left(-B \eta^{\frac{1}{1-\alpha}}\right) d \eta \\
\leq c\left(|\log z|+\int_{0}^{\infty} \log \eta \eta^{\frac{\alpha}{1-\alpha}} \exp \left(-B \eta^{\frac{1}{1-\alpha}}\right) d \eta\right) \leq c(|\log z|+1) .
\end{gathered}
$$

Hence, it follows that

$$
J_{4}(z) \leq J_{4,1}(z)+J_{4,2}(z) \leq c(|\log z|+1) .
$$


If $m \geq 5$, we put $\zeta=\frac{\eta}{z}$ and deduce

$$
\begin{gathered}
J_{m}(z)=\int_{0}^{\infty}(\zeta z)^{1-\frac{m}{2}} \exp \left(-B\left(\frac{1}{\zeta}+(z \zeta)^{\frac{1}{1-\alpha}}\right)\right) z d \zeta \\
\leq c z^{2-\frac{m}{2}} \int_{0}^{\infty} \zeta^{1-\frac{m}{2}} \exp \left(-B \frac{1}{\zeta}\right) d \zeta \leq c z^{2-\frac{m}{2}} .
\end{gathered}
$$

Using (5.8), (5.9), (5.10), we get (5.7). Substituting (5.7) and $z=\frac{|x|^{2}}{t^{\alpha}}$ in (5.6), we obtain (3.19).

\subsection{The development of formula (3.57)}

Estimate (3.24) implies

$$
\left|\left(G *_{2} \Psi\right)(x, t)\right| \leq c|\Psi|_{\mathbb{R}_{T}^{n-1}} \int_{0}^{t} \tau^{\beta-1} d \tau \leq c|\Psi|_{\mathbb{R}_{T}^{n-1}} t^{\beta},
$$

so

$$
\left.\left(G *_{2} \Psi\right)(x, t)\right|_{t=0}=0 .
$$

By definitions (2.5), (2.7) and assumption (3.5), we see

$$
\partial_{t}^{\alpha}\left(G *_{2} \Psi\right)=D_{t}^{\alpha}\left(G *_{2} \Psi\right)=\frac{\partial}{\partial t}\left(I_{t}^{\alpha-1}\left(G *_{2} \Psi\right)\right) .
$$

Now we introduce a sequence

$$
\mathrm{U}_{\varepsilon}(x, t)=\int_{0}^{t-\varepsilon} d \tau \int_{\mathbb{R}^{n-1}} I_{t}^{\alpha-1} G\left(x^{\prime}-y^{\prime}, x_{n}, t-\tau\right) \Psi\left(y^{\prime}, \tau\right) d y^{\prime} .
$$

We need to prove that $\lim _{\varepsilon \rightarrow 0} \frac{\partial}{\partial t} \mathrm{U}_{\varepsilon}$ is equal to the right-hand side of (3.57).

First we obtain

$$
\begin{gathered}
\frac{\partial}{\partial t} \mathrm{U}_{\varepsilon}(x, t)=\int_{-\infty}^{t-\varepsilon} d \tau \int_{\mathbb{R}^{n-1}} D_{t}^{\alpha} G\left(x^{\prime}-y^{\prime}, x_{n}, t-\tau\right) \Psi\left(y^{\prime}, \tau\right) d y^{\prime} \\
+\int_{\mathbb{R}^{n-1}} I_{t}^{\alpha-1} G\left(x^{\prime}-y^{\prime}, x_{n}, \varepsilon\right) \Psi\left(y^{\prime}, \tau-\varepsilon\right) d y^{\prime}
\end{gathered}
$$




$$
=\int_{\varepsilon}^{\infty} d \tau \int_{\mathbb{R}^{n-1}} D_{t}^{\alpha} G\left(x^{\prime}-y^{\prime}, x_{n}, \tau\right)\left(\Psi\left(y^{\prime}, t-\tau\right)-\Psi\left(y^{\prime}, t-\varepsilon\right)\right) d y^{\prime} .
$$

Then we consider the difference between $\frac{\partial}{\partial t} \mathrm{U}_{\varepsilon}$ and the right-hand side of (3.57)

$$
\begin{aligned}
\delta_{\varepsilon}=\frac{\partial}{\partial t} & \mathrm{U}_{\varepsilon}(x, t)-\int_{0}^{\infty} d \tau \int_{\mathbb{R}^{n-1}} D_{t}^{\alpha} G\left(x^{\prime}-y^{\prime}, x_{n}, \tau\right)\left(\Psi\left(y^{\prime}, t-\tau\right)-\Psi\left(y^{\prime}, t\right)\right) d y^{\prime} \\
& =\int_{\varepsilon}^{\infty} d \tau \int_{\mathbb{R}^{n-1}} D_{t}^{\alpha} G\left(x^{\prime}-y^{\prime}, x_{n}, \tau\right)\left(\Psi\left(y^{\prime}, t\right)-\Psi\left(y^{\prime}, t-\varepsilon\right)\right) d y^{\prime} \\
& +\int_{0}^{\varepsilon} d \tau \int_{\mathbb{R}^{n-1}} D_{t}^{\alpha} G\left(x^{\prime}-y^{\prime}, x_{n}, \tau\right)\left(\Psi\left(y^{\prime}, t-\tau\right)-\Psi\left(y^{\prime}, t\right)\right) d y^{\prime}
\end{aligned}
$$

We claim that

$$
\lim _{\varepsilon \rightarrow 0} \delta_{\varepsilon}=0
$$

Indeed, by (3.24), we get

$$
\left|\delta_{\varepsilon}\right| \leq c\langle\Psi\rangle_{t, \mathbb{R}_{T}^{n-1}}^{\left(\frac{1+\theta}{2} \alpha\right)}\left(\varepsilon^{\frac{1+\theta}{2} \alpha} \int_{\varepsilon}^{\infty} \tau^{-1-\beta} d \tau+\int_{0}^{\varepsilon} \tau^{\frac{\theta}{2} \alpha-1} d \tau\right) \leq c\langle\Psi\rangle_{t, \mathbb{R}_{T}^{n-1}}^{\left(\frac{1+\theta}{2} \alpha\right)} \varepsilon^{\frac{\theta}{2} \alpha} .
$$

\section{References}

[1] M. Caputo, Models of Flux in Porous Media with Memory. - Water Resources Research 36 (2000), No. 3, 693-705.

[2] Ph. Clément, S.-O. Londen, and G. Simonett, Quasilinear Evolutionary Equations and Continuous Interpolation Spaces. - J. Diff. Eqs. 196 (2004), No. 2, 418-447.

[3] S.D. Eidelman and A.N. Kochubei, Cauchy Problem for Fractional Diffusion Equations. - J. Diff. Eqs. 199 (2004), 211-255.

[4] H. Engler, Strong Solutions of Quasilinear Integro-Differential Equations with Singular Kernles in Several Space Dimensions. - Electronic J. Diff. Eqs. (1995), No. $2,1-16$.

[5] I.S. Gradshteyn and I.M. Ryzhik, Table of Integrals, Sums, Series and Products. Fizmatgiz, Moscow, 1963. (Russian)

[6] J. Kemppainen, Existence and Uniqueness of the Solution for a Time-Fractional Diffusion Equation with Robin Boundary Condition. Abstract and Applied Analysis 2011, Article ID 321903, 11 pages. 
[7] A.A. Kilbas, Fractional Calculus of the Generalized Wright Functions. - Fractional Calculus and Applied Analysis 8 (2005), No. 2, 113-126.

[8] A.A. Kilbas, H.M. Srivastava, and J.J. Trujillo, Theory and Applications of Fractional Differential Equations. North Holland, Mathematical studies, 204, Elsevier Science B.V., Amsterdam, 2006.

[9] A.N. Kochubei, Fractional-Order Diffusion. - Diff. Eqs. 26 (1990), 485-492.

[10] A.N. Kochubei, Fractional Parabolic Systems. - Potential Analysis 37 (2012), $1-30$.

[11] M. Krasnoschok and N. Vasylyieva, On a Solvability of Nonlinear Fractional Reaction-Diffusion System in the Hölder Spaces. - Nonlinear Studies 20 (2013), No. 4, 591-621.

[12] O.A. Ladyzhenskaya, V.A. Solonnikov, and N.N. Ural'tseva, Linear and Quasilinear Parabolic Equations. Transl. Math. Monogr. 23 AMS, Providence, RI, 1968.

[13] H.P. Lopushans'ka and A.O. Lopushans'kyi, Space-Time Fractional Cauchy Problem in Spaces of Generalized Functions. - Ukr. Math. J. 64 (2013), No. 8, 12151230 .

[14] R. Metzler and J. Klafter, The Random Walk's Guide to Anomalous Diffusion: a Fractional Dynamics Approach. - Phys. Rep. 339 (2000), 1-77.

[15] G.M. Mophou and G.M. N'Guérékata, On a Class of Fractional Differential Equations in a Sobolev Space. - Applicable Analysis 91 (2012), No. 1, 15-34.

[16] A.M. Nahushev, Fractional Calculus and Its Applications. Nauka, Moscow, 2003. (Russian)

[17] I. Podlubny, Fracrional Differential Equations. Academic Press, San-Diego, 1999.

[18] R. Ponce, Hölder Continuous Solutions for Fractional Differential Equations and Maximal Regularity. — J. Diff. Eqs. 255 (2013), 3284-3304.

[19] A.V. Pskhu, A Fundamental Solution for a Fractional Diffusion Wave Equation. Izvestia RAN 73 (2009), 141-181. (Russian)

[20] A.V. Pskhu, Partial Differential Equations of the Fractional Order. Nauka, Moscow, 2005. (Russian)

[21] K. Sakamoto and M. Yamamoto, Initial Value Boundary Value Problems for Fractional Diffusion-Wave Equations and Applications to Some Inverse Problems. - J. Math. Anal. Appl. 382 (2011), 426-447.

[22] W.R. Schneider and W. Wyss, Fractional Diffusion and Wave Equations. - J. Math. Phys. 30 (1989), 134-144.

[23] V. Tarasov, Fractional Dynamics: Applications of Fractional Calculus to Dynamics of Particles. Fields and Media. Higher Education Press, Beijing and Springer-Verlag, Berlin, Heidelberg, 2010.

[24] R. Zacher, Quasilinear Parabolic Problems with Nonlinear Boundary Conditions. Ph. D. Thesis, Martin-Luther-Universität, Halle-Wittenberg (2003). 